\title{
Amphiphiles Self-Assembly: Basic Concepts and Future Perspectives of Supramolecular Approaches
}

\author{
Domenico Lombardo, ${ }^{1}$ Mikhail A. Kiselev, ${ }^{2}$ Salvatore Magazù, ${ }^{3}$ and Pietro Calandra ${ }^{4}$ \\ ${ }^{1}$ Consiglio Nazionale delle Ricerche (CNR), Istituto per i Processi Chimico-Fisici (IPCF), Viale F.S. D’Alcontres, No. 37, \\ 98158 Messina, Italy \\ ${ }^{2}$ Frank Laboratory of Neutron Physics, Joint Institute for Nuclear Research, Ulica Joliot-Curie 6, Dubna, Moscow 141980, Russia \\ ${ }^{3}$ Dipartimento di Fisica e Scienze della Terra, Università di Messina, Viale F.S. D’Alcontres, No. 31, 98158 Messina, Italy \\ ${ }^{4}$ Consiglio Nazionale delle Ricerche (CNR), Istituto per lo Studio dei Materiali Nanostrutturati (ISMN), Via Salaria km 29.300, \\ Monterotondo Stazione, 00015 Roma, Italy
}

Correspondence should be addressed to Domenico Lombardo; lombardo@me.cnr.it

Received 5 May 2015; Accepted 27 September 2015

Academic Editor: Ram N. P. Choudhary

Copyright $\odot 2015$ Domenico Lombardo et al. This is an open access article distributed under the Creative Commons Attribution License, which permits unrestricted use, distribution, and reproduction in any medium, provided the original work is properly cited.

\begin{abstract}
Amphiphiles are synthetic or natural molecules with the ability to self-assemble into a wide variety of structures including micelles, vesicles, nanotubes, nanofibers, and lamellae. Self-assembly processes of amphiphiles have been widely used to mimic biological systems, such as assembly of lipids and proteins, while their integrated actions allow the performance of highly specific cellular functions which has paved a way for bottom-up bionanotechnology. While amphiphiles self-assembly has attracted considerable attention for decades due to their extensive applications in material science, drug and gene delivery, recent developments in nanoscience stimulated the combination of the simple approaches of amphiphile assembly with the advanced concept of supramolecular self-assembly for the development of more complex, hierarchical nanostructures. Introduction of stimulus responsive supramolecular amphiphile assembly-disassembly processes provides particularly novel approaches for impacting bionanotechnology applications. Leading examples of these novel self-assembly processes can be found, in fact, in biosystems where assemblies of different amphiphilic macrocomponents and their integrated actions allow the performance of highly specific biological functions. In this perspective, we summarize in this tutorial review the basic concept and recent research on self-assembly of traditional amphiphilic molecules (such as surfactants, amphiphile-like polymers, or lipids) and more recent concepts of supramolecular amphiphiles assembly which have become increasingly important in emerging nanotechnology.
\end{abstract}

\section{Introduction}

Self-assembly processes involving amphiphilic macromolecules provide unique and new opportunities for designing novel materials for advanced application in nanotechnology [1-3]. The thermodynamic incompatibility between the different blocks causes a spatial organization into ordered morphologies on the nanoscale with the production of novel structural features, as demonstrated by recent studies [3, 4]. Leading examples can be found in biosystems where assemblies of different amphiphilic macromolecular components and their integrated actions allow the performance of highly specific cellular functions [3-5]. In the first part of this review we present a tutorial introduction to the basic aspect of traditional, head/tail(s) type, amphiphiles whose aggregation is driven by soft interactions such as hydrogen bonds and steric effects and hydrophobic and electrostatic interaction. Moreover, we highlight important examples where complex processes such as the structure modulation and control of morphology by other structure directing interactions can stimulate advanced application in materials science as well as in biological and medicinal chemistry. Finally, we provide insight into the novel structural features obtained by the precise tailoring of chemical structures and the efficient use of noncovalent forces for the introduction of chirality, signal processing, and recognition processes. The reversibility of 


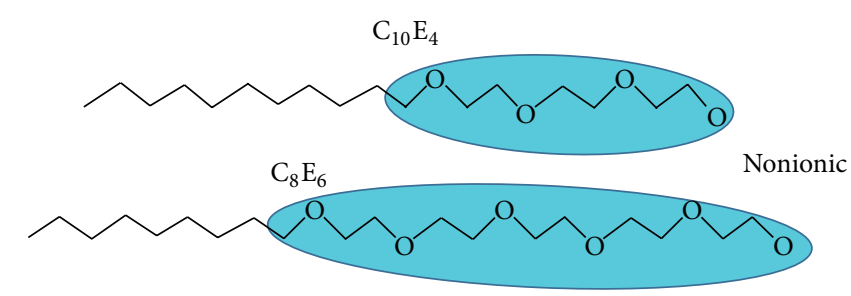

Polyoxyethylene alkyl ethers $\left(\mathrm{C}_{n} \mathrm{E}_{m}\right)$

(a)
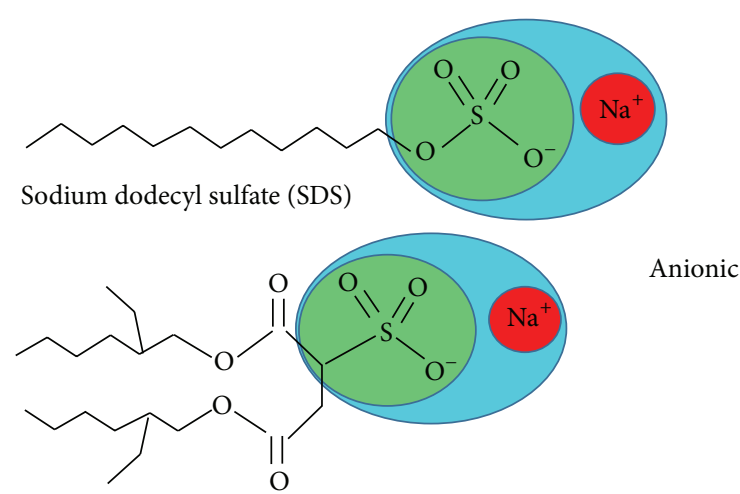

Sodium bis(2-ethylhexyl) sulfosuccinate (AOT)

(b)

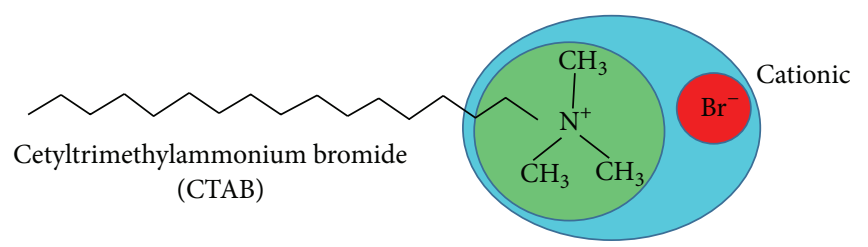

(c)

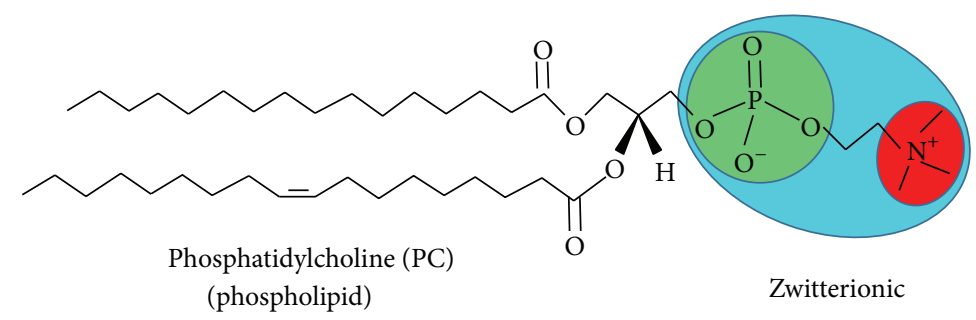

(d)

Figure 1: Example of most common nonionic (a), anionic (b), cationic (c), and zwitterionic (d) amphiphilic molecule.

noncovalent interactions allows dynamic switching of nanostructures morphology and functions in response to various external stimuli which further provides a flexible platform for the design and fabrication of smart amphiphilic nanomaterials and functional supramolecular devices.

\section{Characteristic and Basic Properties of Amphiphiles}

Amphiphiles are compounds possessing both hydrophilic (water-loving) and lipophilic (fat-loving) or water-hating components. In conventional head/tail(s) amphiphiles the lipophilic part consists generally of a long (saturated or unsaturated) hydrocarbon chain, while the hydrophilic head can be either nonionic or ionic. Nonionic surfactants have either polyether or polyhydroxyl units as the hydrophilic group. A large variety of conventional nonionic surfactants consist generally of a hydrophilic poly(ethylene oxide) chain, often called ethoxylates, connected with a hydrophobic alkyl chain, and are generally used in cleaning applications with anionic surfactants. For example, polyoxyethylene alkyl ethers, $\mathrm{C}_{n} \mathrm{E}_{m}$, are nonionic surfactants made of $m$ hydrophilic oxyethylene units and an alkyl chain with $n$ methylene groups
(Figure 1(a)). Anionic surfactants, which are widely used as detergents and soap for cleaning processes, consist generally of negatively charged headgroups and positively charged counterions (such as sodium, potassium, or ammonium ions). Carboxylate, sulfate, sulphonate, and phosphate are the commonly used polar groups (Figure 1(b)). Cationic surfactants consist of positively charged headgroups such as a quaternary ammonium and a halide ion as a counterion. Cetyltrimethylammonium bromide (CTAB) and sodium bis(2ethylhexyl) sulfosuccinate (AOT) are the most employed cationic amphiphiles (Figures 1(b) and 1(c)). Finally in zwitterionic amphiphiles the headgroups possess both a positive and negative charge, as it happens, for example, in the vesicleforming phospholipid phosphatidylcholine (Figure 1(d)). For example, if zwitterions contain a carboxylate and a protonated ammonium ion, it may behave as an anion (at high $\mathrm{pH}$ ) or a cation (at low $\mathrm{pH}$ ) assuming then an amphoteric character. Some traditional amphiphilic molecules are reported in Figure 1.

Due to their amphiphilicity (or surface activity), the amphiphiles polar headgroup interacts with the water while the nonpolar lipophilic chain will migrate above the interface (either in the air or in a nonpolar liquid). In this case the disruption of the cohesive energy at the interface favors 


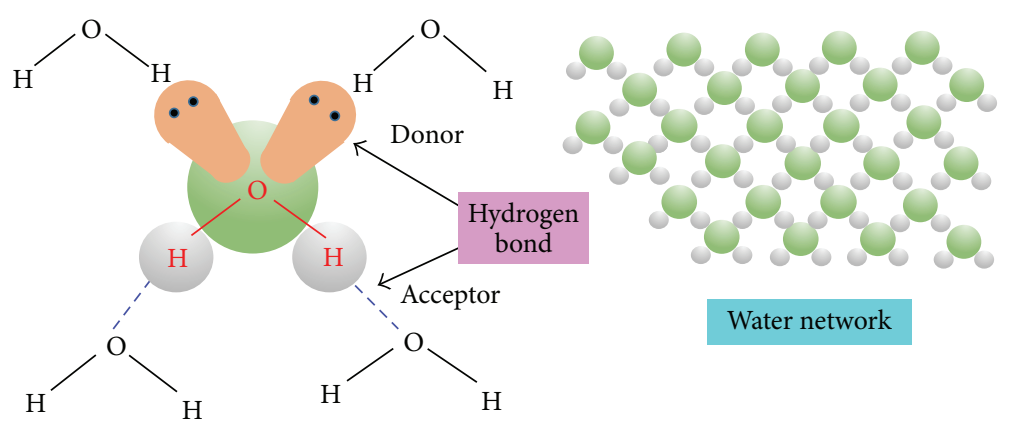

FigURE 2: Schematic representation of polarity of water molecules and hydrogen bond in water.

a microphase separation between the selective solvent and the dispersed phase of the amphiphile with the formation of many smaller closed interfaces or micelles-like aggregates. Due to their ability to reduce the interfacial tension amphiphilic molecules are often called surfactants (i.e., surface active agents). For this reason amphiphiles play an important role as emulsifiers, detergents, dispersants, and wetting and foaming agents in several applications $[6,7]$.

\section{Soft Interaction in Amphiphiles Self-Assembly}

Despite the weakness of the forces involved in amphiphiles self-assembly, the relevant number of these soft interactions will produce an overall effect that is strong enough to hold different amphiphile molecules together as well as to ensure their stability in solution $[8,9]$. Moreover, the weakness of the involved interactions makes the structure more flexible enabling the system to withstand minor perturbation while preserving the reversibility of the self-assembled structure. The main weak (noncovalent) forces acting in the amphiphiles self-assembly are hydrogen bonding, hydrophobic effects, electrostatic interaction, and van der Waals forces. The strength of those soft interactions, which are much less intense than the covalent bonds $\left(400 \mathrm{~kJ} \mathrm{~mol}^{-1}\right)$, varies from less than $5 \mathrm{~kJ} \mathrm{~mol}^{-1}$ for van der Waals forces to up to a maximum $120 \mathrm{~kJ} \mathrm{~mol}^{-1}$ for hydrogen bond (or up to 400 for metalligand interactions). A summary of those soft interactions is presented in Table 1.

Stability in solution of the amphiphiles within the aggregates is given both to the hydration of the hydrophilic (polar) headgroups and to the insertion of the hydrophobic (apolar) tail(s) in the solvent. The first is an enthalpic gain in solvation due to hydrogen bond formation while the second, called the hydrophobic effect, is a gain in entropy of the bulk water.

Hydrogen bonds are essential for important functions in biological systems as they are strong enough to bind biomolecules together but weak enough, when necessary, to be broken inside living cells. Biomolecules, in fact, have $H$-bond acceptors and donors within them due to the presence of molecular dipoles of hydrogens $(\mathrm{H})$ atoms bonded with electronegative atoms (like oxygen, nitrogen, or fluorine). In water the nonbonding electrons are the $H$-bond acceptors and the hydrogen atoms are the $\mathrm{H}$-bond donors. As shown in
TABLE 1: Strength of the main noncovalent interaction involved in amphiphiles self-assembly.

\begin{tabular}{lc}
\hline Bonding and interaction type & $\mathrm{kJ} / \mathrm{mol}$ \\
\hline Covalent bond & $100-400$ \\
Ion-ion/ion-dipole/dipole-dipole & $200-300 / 50-200 / 5-50$ \\
Hydrogen bond & $4-120$ \\
Cation- $\mathrm{n}(\pi)$ interaction & $5-80$ \\
$\pi-\pi$ interaction & $0-50$ \\
van der Waals interaction & $(<5 \mathrm{~kJ} / \mathrm{mol})$ \\
Hydrophobic effects & Entropy \\
Metal-ligand & $0-400$ \\
\hline
\end{tabular}

Figure 2, the polarity of water molecules results in the attraction of the negative (oxygen) portion and positive (hydrogen) partial charges and is at the basis for the water hydrogen bonding which have many important implications on the properties of water and its relevant functions in biological systems [10-13].

Together with the hydrogen bond, the hydrophobic effect is the second main driving force of amphiphile self-assembly into various supramolecular structures. The hydrophobic effect plays an important role in many soft matter systems as it regulates the tendency of nonpolar (hydrophobic) molecules to self-aggregate $[14,15]$. When a hydrophobic compound is inserted in water we observe the disruption of the $\mathrm{H}$-bonding water network that favors a rearrangement of the water molecules around the nonpolar molecules. When different nonpolar molecules are dissolved in water the disruption of the H-bonding water network favors the creation of larger cavities to accommodate an assembly of nonpolar (solute) molecules. In this case water molecules structures that are distorted by the presence of the hydrophobe will make new hydrogen bonds thus inducing an ice-like cage structure (called a clathrate) around the hydrophobic molecules $[15,16]$. This process corresponds to an effective mutual attraction between the nonpolar molecules in water (hydrophobic interaction), while entropically more favorable aggregated structures are generated to minimize the disruption of the water structure.

The hydrophobic effect plays a crucial role not only in the formation of amphiphilic micellar aggregates but also for a wide range of other biological processes such as protein 


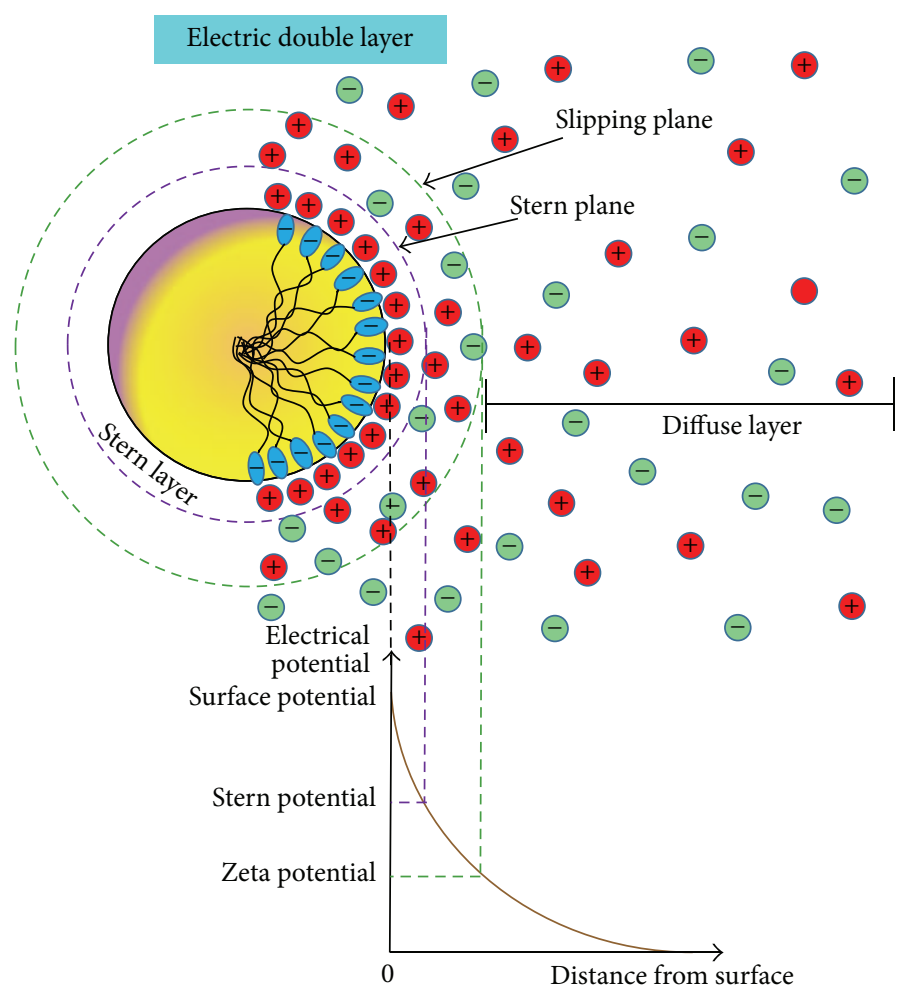

FIGURE 3: Schematic representation of the electrical double layer of self-assembled charged amphiphilic molecules.

folding or drug delivery in biomembranes. Synergistic effects of hydrophobic and hydrogen bond interactions are important in keeping a protein alive and biologically active through the folding processes which allow the protein to decrease in surface thus reducing the undesired interactions with water [12-18].

Another important interaction, which is mainly connected with ionic amphiphiles self-assembly and stability in solution, is represented by the Derjaguin, Landau, Vervey, and Overbeek (DLVO) theory of charged colloids $[19,20]$. This theory represents the cornerstone to rationalize forces between charged colloids (or charged amphiphiles) at interfaces and to explain their aggregation behavior in solution. DLVO theory assumes that the interaction in solution between charged colloids can be approximated by two principal contributions, namely, the van der Waals and double layer interactions [20-22]. The van der Waals interaction is composed by a short-range repulsive (hard sphere) force and short-range attractive London dispersion force generated by a temporary (short-lived) dipole induced by a polarization of the electron distribution of an adjacent atom. The magnitude of this short-range attractive interaction is generally approximated by the simple "6-12 Lennard-Jones potential" $V_{\mathrm{VdW}}=\left(A / r^{6}-B / r^{12}\right)[21]$. For example, hydrocarbons which do not carry any charges (and have no permanent dipole) are dominated by van der Waals interactions. Those interactions are extremely important in biomembranes where the phospholipids are tightly packed in aqueous medium $[5,23]$. The double layer interaction originates from the dissociation of the amphiphile surface groups or adsorption of charged molecules (such as polyelectrolyte) from the solution. As described in Figure 3 the electrical double layer (EDL) consists of (immobile) layer of ions strongly bound to the charged surface (Stern layer) and an adjacent region of loosely associated mobile ions (diffuse layer). The total electrical double layer due to the formation of the counterion layers results in electrostatic screening of the aggregate wall charge and minimizes the Gibbs free energy of EDL formation. In a first approximation the thickness of the diffuse electric double layer is represented as the inverse of the Debye-Huckel screening constant $\kappa=\left(8 \pi e^{2} N_{a} I / \varepsilon K_{B} T \times 10^{3}\right)^{1 / 2}$ which is determined, at a given temperature $T$, by the ionic strength $I$ of the solvent (in mol/L) (where $e$ is the unit of electron charge, $K_{B}$ is the Boltzmann constant, and $N_{a}$ is the Avogadro number).

\section{Amphiphiles Self-Assembly and Aggregates Formation}

Self-assembly is a process by which disordered building blocks governed by specific interblocks mutual interaction form an ordered structure through a spontaneous organization. The building blocks are not necessarily amphiphile molecules but can span a wide range of macromolecular nano- and mesoscopic structures with different chemical compositions, shapes, and functionalities. For example, the polypeptide chains folding into proteins or conformational changes of nucleic acids into their different functional forms are relevant examples of self-assembly processes in biological systems. The main requirement to have a favorable spontaneous organization toward a self-assembled structure is the minimum 
energy configuration at equilibrium. More specifically the generated self-assembled structure has a higher order (lower entropy) than the isolated components, while its surroundings present generally a more disordered configuration (higher entropy). In this respect from the thermodynamic point of view the self-association and micellar formation of amphiphilic molecules are driven by the competition between interfacial energy of the micelle core with solvent and the conformational distortion energy of the soluble chains emanating from the core. According to the closed association model the detected critical micellar concentration (CMC) can be used to obtain information on the thermodynamic parameters of the micellization process $[1,2,6]$. The standard Gibbs free energy change $\Delta G$ for the transfer of $1 \mathrm{~mol}$ of amphiphile from solution to the micellar phase (so-called free energy of micellization) can be expressed as a function of the absolute temperature $T$ and the $\mathrm{CMC}$ molar fraction $X_{\mathrm{CMC}}$ and can be approximated by $\Delta G \approx R T \ln \left(X_{\mathrm{CMC}}\right)$ (where $R$ is the ideal gas constant) [6-9]. Amphiphiles self-assembly can be demonstrated experimentally through the measurement of a discontinuity, usually associated with the formation of micelles, in the physical property at a given concentration (critical micelle concentration-CMC) and temperature (critical micelle temperature-CMT). Together with theoretical modeling and simulation approaches $[1,2,24]$, useful physical quantities for detecting the $\mathrm{CMC}$ are the equivalent conductivity, osmotic pressure, surface tension, and fluorescence of a chromophore added to the solution. Some of these quantities are shown in Figure 4.

For commonly used surfactants the CMC is typically less than about $0.01 \mathrm{M}$ (e.g., for sodium dodecyl sulfate: $\mathrm{CMC}=$ $0.008 \mathrm{M}$ ). It is worth mentioning that different experimental $[25,26]$ and theoretical [27] investigations have provided indication of an amphiphiles premicellar aggregation with the appearance of aggregates at concentrations four times lower than the macroscopically determined CMC as indicated by fluorescence correlation spectroscopy experiment [25]. Combining thermodynamic model with reaction rate theory it has been demonstrated that, over most of the metastable concentration range, the premicellar aggregates have macroscopic lifetimes and small polydispersity [27]. Finally, it is important to notice that the micellar aggregates should not be considered as "permanent" frozen structures, but they are rather thermodynamic equilibrium systems with a dynamic structure in which the unimers exchange rapidly with the bulk solution. Lifetime of molecule in a small micelle is between $10^{-5}$ and $10^{-3} \mathrm{~s}$.

\section{Amphiphile Packing Factors and Aggregates Morphologies}

The shape and size of given micellar aggregates depend on the molecular geometry of its component surfactant molecules and the solution conditions such as surfactant concentration, temperature, $\mathrm{pH}$, and ionic strength. Control over the shapes gives a possibility to develop and manipulate nanostructures architecture. In this way, exciting functional systems are

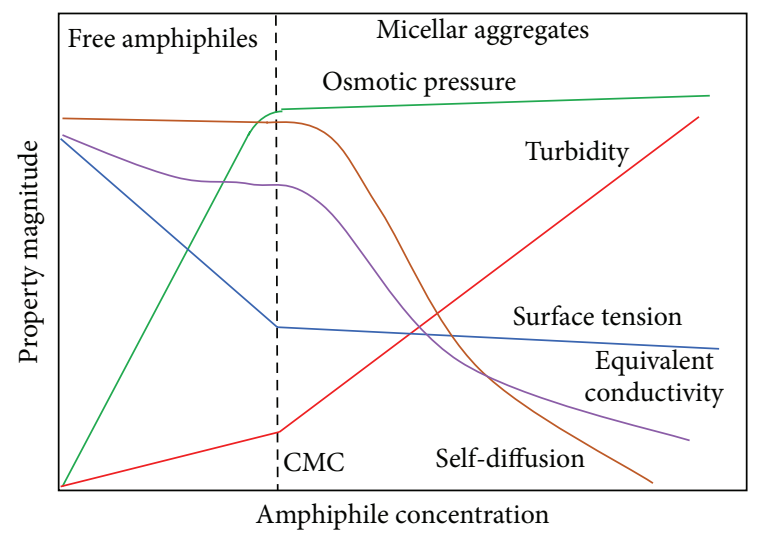

FIgURE 4: Behavior of some physical properties and their discontinuity associated with the formation of micelles at the critical micelle concentration (CMC) for amphiphiles in a selective solvent.

developed which can find a wide range of technological applications in different fields of science. Preliminary estimates of shape and size can be made with the analysis of the critical packing factor $\left(C_{\mathrm{pp}}\right)$. According to Israelachvili $[1,8]$ the structure of the aggregate can be predicted from the critical packing parameter $C_{\mathrm{pp}}=V_{0} / A_{\text {mic }} l_{c}$, where $V_{0}$ is the effective volume occupied by hydrophobic chains in the aggregate core, $l_{c}$ is the maximum effective length (critical chain length), and $A_{\text {mic }}$ is the effective hydrophilic headgroup surface area at the aggregate-solution interface. With the increment of $C_{\mathrm{pp}}$ value, the structure of aggregates can be spherical $\left(C_{\mathrm{pp}} \leq 1 / 3\right)$, cylindrical $\left(1 / 3 \leq C_{\mathrm{pp}} \leq 1 / 2\right)$, and lamellar $\left(C_{\mathrm{pp}}=1\right)$. For $1 / 2 \leq C_{\mathrm{pp}} \leq 1$ vesicles are generally generated, which correspond to spherical (or ellipsoidal) closed amphiphile bilayer structures with an internal cavity containing the dispersion solution $[1,6]$. A summary of the aggregate structures that can be predicted from the critical packing parameter $C_{\mathrm{pp}}$ is reported in Figure 5.

Typical vesicles are formed by synthetic or natural phospholipids (liposomes) and are generally composed of one hydrophilic head and two hydrophobic tails $[4,5]$. When phospholipids are dispersed in water, they tend to aggregate spontaneously to form bilayers, which resemble the types of structures they form in biological membranes. Bilayer vesicles are generally metastable structures in aqueous solution and, depending on the conditions of preparation (i.e., stirring, sonication, and extrusion), some energy is required to dissolve the amphiphile in water and to induce selfaggregation. In such cases the produced vesicles, with typical sizes ranging from $10 \mathrm{~nm}$ to $10 \mu \mathrm{m}$, may contain one (unilamellar) or more (multilamellar) concentric bilayer surfaces in an onion-like structure (hydrated multilayers) [28-30].

For packing parameters larger than one $\left(C_{\mathrm{pp}}>1\right)$ inverse micelles can form. In this case, hydrophobic chains radiate away from centrally aggregated headgroups that surround the water solvent. Inverse aggregates also form in isotropic and thermodynamically stable ternary systems such as microemulsions, where two immiscible phases (water and "oil") are 

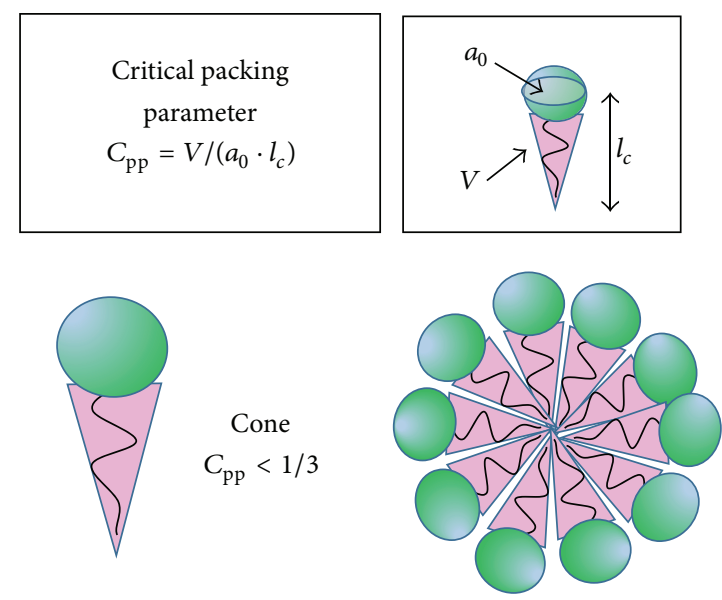

Spherical micelles
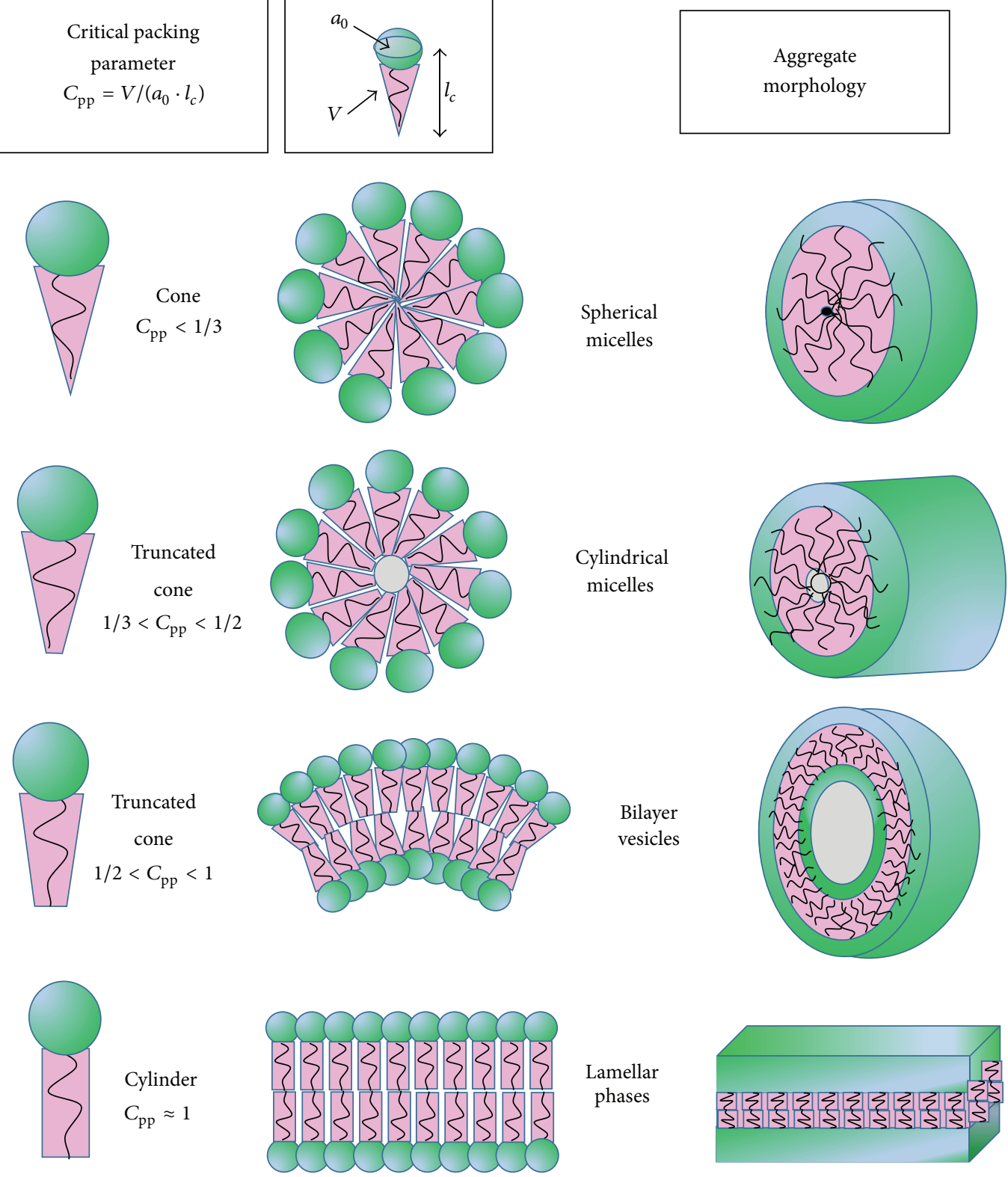

Lamellar
phases
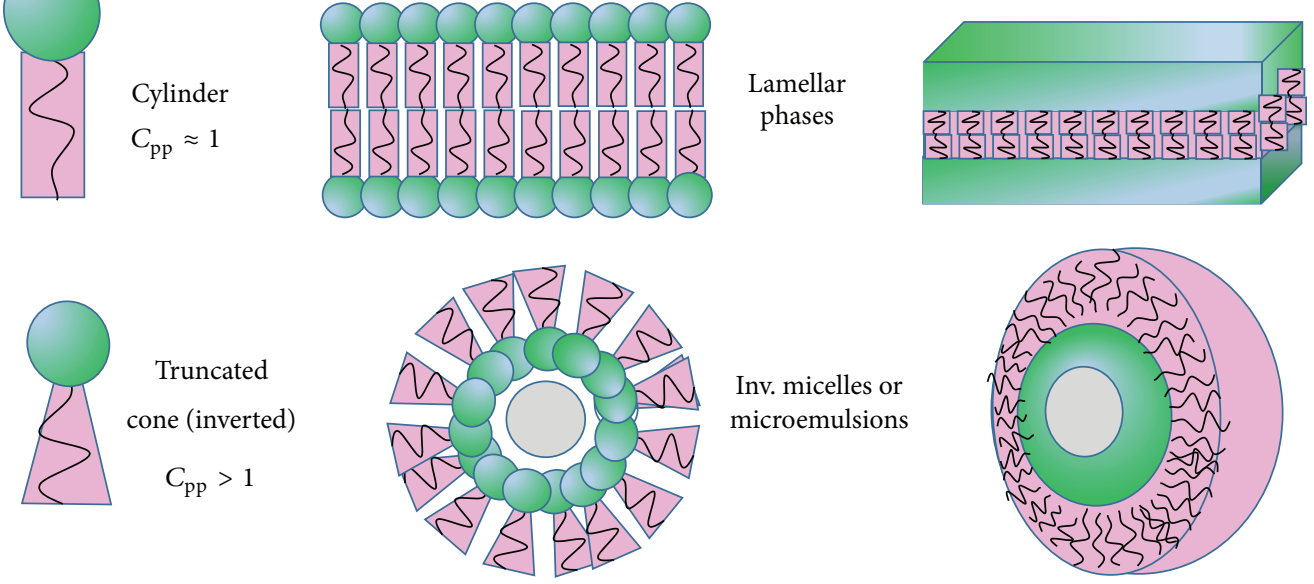

FIGURE 5: Amphiphile shape factors and summary of the aggregate structures that can be predicted from the critical packing parameter $C_{\mathrm{pp}}$.

present with a surfactant, such as the AOT amphiphile presented in Figure 1(d). The surfactant molecules, often in combination with a cosurfactant, form a monolayer at the oilwater interface, with the surfactant hydrophobic tails dissolved in the oil phase while the hydrophilic headgroups stay in the aqueous phase. The basic types of microemulsions are the "direct" oil-in-water (o/w), the "reversed" of water-inoil (w/o), and the bicontinuous, while the microemulsions droplet radii are usually in the range of $5-50 \mathrm{~nm}[31,32]$. It is worth pointing out that the precise description of aggregate 
morphology for amphiphiles with more complex topology is more difficult to rationalize due to the different involved intermolecular interactions and complex synergistic effects present $[33,34]$.

\section{Phase Diagrams and Lyotropic Liquid Crystal Structures of Amphiphiles}

As previously stated the amount of amphiphilic molecules influences the morphology of the self-assembled structures. While at very low concentration the molecules will be dispersed randomly without any ordering, at the higher amphiphile concentrations the self-assembled nanostructures can order themselves in much the same way as thermotropic liquid crystals do. The major prerequisite for the appearance of liquid crystal phases is anisotropy. While for thermotropic liquid crystals no solvent is required, for lyotropic liquid crystals (LLC) formed by amphiphilic molecules in selective solvents the anisotropy is based mainly on the different solubility properties of the different ends of the amphiphiles and a subtle balance of intermolecular interactions [3537]. Different classes of lyotropic liquid crystals phases of surfactant systems have been extensively investigated over the whole concentration range while different order parameters of their structures have been classified by means of X-ray diffraction patterns and chemical shift splitting in nuclear magnetic resonance (NMR) spectra [37-39].

In Figure 6 a typical amphiphile LLC phase diagram is presented. Surfactants dissolved in water generally present a Krafft point, defined as the temperature $\left(T_{K}\right)$ below which amphiphile crystals are insoluble in water. As indicated in the right side of Figure 6, at extremely high concentrations of amphiphile reversed (or inverted) LLC phases are often observed. Generally, more complex phase behavior is observed in nonionic amphiphiles [40]. For instance, in polyoxyethylene- (EO-) based nonionic surfactant a decrease in the EO headgroups hydration at the higher temperature causes an effective attraction between adjacent micelles. In this case the appearance of a cloud point indicates the temperature (and concentration) at which phase separation occurs, while the solution becomes turbid (cloudy) due to the formation of an amphiphile-rich droplets phase. The lowest cloud point is referred to as the lowest critical solution temperature (LCST). Below the LCST surfactants dissolve in solution and above LCST the nonionic amphiphiles separate from the water solution and new two isotropic liquid phases are formed in aqueous solution. Complex structural and dynamic features as well as collective phenomena involving a large number of macromolecules are the main factors influencing amphiphile systems near the cloud point [40]. In Figure 7 a schematic representation of a typical progression of phases as a function of concentration for an amphiphile dissolved in a selective solvent is presented. The cubic LLC phase consists of a cubic arrangement of molecular aggregates of "normal" micelles in a water continuous ( $\mathrm{I}_{1}$ phase). The reversed micelles phase ( $\mathrm{I}_{2}$ phase) generally lies between the reversed hexagonal $\left(\mathrm{H}_{2}\right)$ and reversed micellar $\left(\mathrm{L}_{2}\right)$ phases. Cubic phases are more viscous than the corresponding hexagonal or lamellar phases.

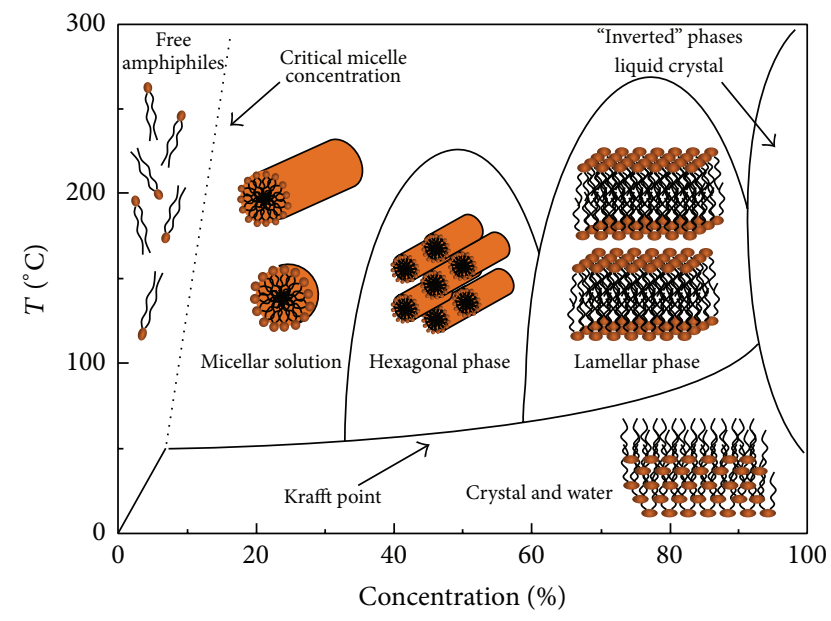

FIGURE 6: Typical lyotropic liquid crystalline (LLC) phase diagram of amphiphiles dissolved in a selective solvent.

In the hexagonal LLC phases amphiphiles form (indefinite length) micellar cylinders that arrange themselves into a roughly hexagonal lattice, whose spacing depends upon the relative amounts of water and surfactant. There are two main types of hexagonal phases, the "direct" hexagonal phase $\left(\mathrm{H}_{1}\right)$ and the reversed hexagonal phase $\left(\mathrm{H}_{2}\right)$ where the micellar cylinders are reversed with the hydrophobic chains radiating outwards from the cylinders. Hexagonal LLC phases typically contain $30 \%$ to $60 \%$ water by weight and despite this high water content the phase is very viscous. For some systems between the hexagonal and lamellar phases, a highly viscous dense (cubic lattice) isotropic phase may exist wherein spheres are formed. These spheres may also be connected to one another, forming a bicontinuous cubic phase $\left(\mathrm{V}_{1}\right.$ and $\mathrm{V}_{2}$ ). Finally the lamellar LCC phase $\left(\mathrm{L}_{\alpha}\right)$ structures consist of a layered arrangement of amphiphiles and are generally less viscous than the hexagonal LLC ones, as the parallel layers slide over each other with relative ease during shear.

The lamellar arrangement represents a particularly important configuration of the liquid crystalline structure of biological membranes $[4,5]$. In cell plasma biomembranes, formed prevalently by liquid crystalline phospholipids bilayers, the hydrocarbon chains are not rigid and a gel phase transition takes place at a given temperature $T_{\mathrm{GEL}}$. Below $T_{\mathrm{GEL}}$ the headgroups become arranged in a more ordered manner with a more straight conformation of the hydrocarbon chains. The liquidity of biomembranes facilitates the movement of macromolecules present within the phospholipids matrix $[4,5]$, such as membrane proteins, while the interactions of proteins with phospholipids molecules determine how the sequence of amino acids in a protein is folded, which in turn affects the functioning of the protein.

At very high amphiphile concentration the amphiphilic molecules arrange themselves to maximize their polar-polar and apolar-apolar interactions while minimizing the steric hindrance. The low content of water (or any other molecules that acted as "solvent" in previous cases) makes the structure more rigid and a new region, characterized by liquid crystal 


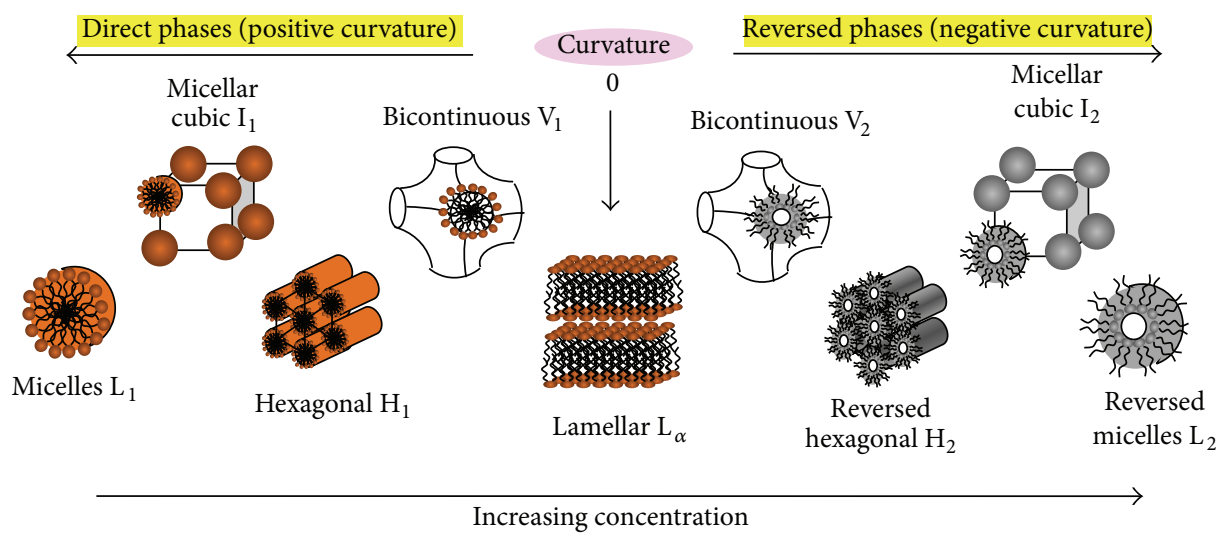

FIGURE 7: Schematic representation of a typical progression of phases as a function of concentration for an amphiphile dissolved in a selective solvent.

phases, is then entered (see right part of Figure 6) [38-41]. The dynamics are not entirely frozen and the amphiphiles form strong templating cages, while the remaining solvent molecules are confined in region with the same polarity [41]. In the extreme situation of pure amphiphiles, although most of them are solid due to their ionic character, nonionic amphiphiles still present interesting examples. In this case amphiphile in the pure and liquid state generally has structures and dynamics typical of structured and glass forming liquids. The opportune mixture of different amphiphiles can trigger therefore the arising of striking emerging properties that can be used for specific technological applications. For example, the high proton conductivity of about $10^{-3} \mathrm{~S} \mathrm{~cm}^{-1}$ at $150^{\circ} \mathrm{C}$ under anhydrous condition in self-assembled acidbase composites formed by the acidic surfactant monododecyl phosphate and the basic surfactant 2-undecylimidazole molecules has been explained in terms of two-dimensional proton-conducting pathways within the polar domains of highly ordered lamellar structures [41], an effect that has been obtained even at room temperature in octanoic acid/bis(2ethylhexyl)amine mixtures. The liquid crystal-like structure in mixtures of pure amphiphiles can give peculiar structural properties that give exotic behavior, especially due to the interplay between local molecular rearrangement and partially/selectively arrested dynamics, like 1D molecular diffusion, anti-Arrhenian behavior of conductivity, and so forth [39-42].

\section{Beyond the Conventional Amphiphiles}

Beyond the more common micelles, vesicles, and liquid crystalline mesomorphic phases, amphiphilic molecules can hierarchically self-assemble in a rich variety of more organized nanostructures such as fibers, ribbons, helices, super helices, and tubes, which have attracted the interest of supramolecular chemists. With the advent of novel synthetized systems containing multiple functional end-groups, it is more difficult to predict what form an assembling system will take even if the basic supramolecular modes of interaction are known. In this case, a wide range of formations is available simply by varying the balance of hydrophilic and hydrophobic components, while recent studies have revealed that chirality may play a critical role in controlling the shape of selfassembled nanostructures.

7.1. Gemini and Bola Amphiphile. Gemini and Bola amphiphiles are two examples of macromolecular amphiphile topology different from the conventional head/tail(s) surfactants. Both Gemini and Bola amphiphiles are good candidate for a wide range of advanced applications such as dispersing, foaming, solubilization, and drug and gene delivery.

Gemini amphiphile (Figure 8) contains two hydrophilic headgroups linked by a spacer and two hydrophobic tails. The two closer hydrophobic chains give to Gemini amphiphiles a more compact molecular packing configuration that induces sensitively low surface tensions. This class of compounds shows intriguing properties such as submicellar aggregation or formation of thread-like micelles. Moreover they generally have a critical micelle concentration (CMC) which is up to two orders of magnitude lower than the CMCs of corresponding single chain surfactants and good solubilization properties [43]. Bola amphiphiles are amphiphilic macromolecules containing two hydrophilic water-soluble groups placed at both ends of a hydrophobic skeleton (generally one or more alkyl chains). The presence of a second headgroup yields a higher solubility in water, an increase in the CMC value, and a decrease in aggregation number with respect to traditional single-headed amphiphiles. Together with the classical spheres, cylinders, disks, and vesicles type aggregates, Bola amphiphiles are also known to form helical structures, which can form microtubular self-assemblies [44].

\subsection{Organized Nanostructure: Micellar Fibers and Distorted} Bilayer-Based Aggregates Morphologies. Most of the novel morphologies of assemblies result from the combination of multiple weak interactions between amphiphilic macromolecules possessing specific packing properties and the experimental conditions of nanostructures self-assembly. On the other hand the prediction and control over the final morphology remain a difficult task while clear design rules have not been unambiguously defined. For example, a recent synthesis approach combines the anisotropic micellization process of 

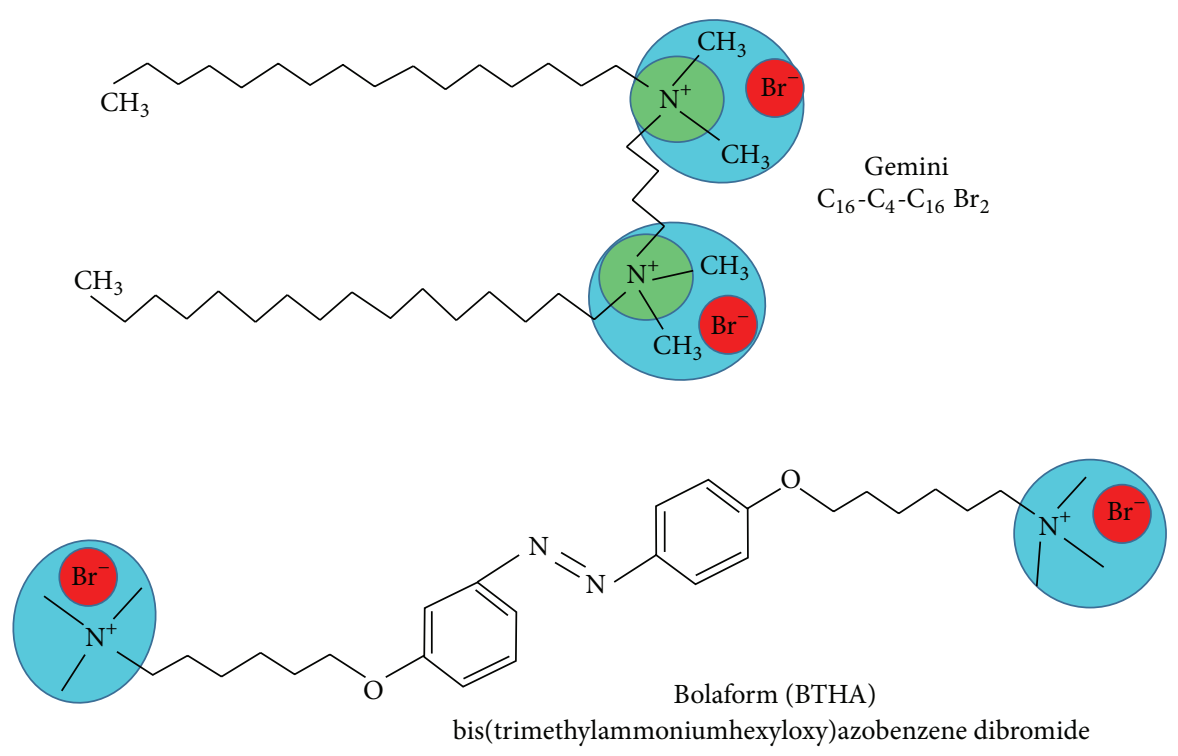

FIGURE 8: Schematic representation of Gemini and Bola amphiphiles.

amphiphilic crystalline-coil block copolymer in water and reassembly effects during single spinneret electrospinning [45]. The resulting core-shell micellar nanofibers structure was composed of a polyvinyl alcohol (PVA) shell and polyethylene glycol-block-poly( $p$-dioxanone) (PEG- $b$-PPDO) as crystallizable core. Recent researches have revealed that chirality plays a crucial role in controlling the shape of selfassembled bilayer sheet-based aggregates such as helicoids, tubules, and spiral ribbons morphologies. With the aim of explaining what influences the formation of curved aggregates different chirality-based theories have been proposed based on chiral self-assembly models [46-48]. According to these theories some chiral constraints in the systems force macromolecules to self-assemble in a nonparallel arrangement (i.e., with nonzero angle with respect to their nearest neighbours) thus generating a preferred orientation in the structure of the bilayer. As a consequence the twisting of the bilayer (distorted bilayers) induces the formation of hollow cylinders and an initial fibrous morphology [46-48].

7.3. Bioinspired Peptide Amphiphiles. Bioinspired amphiphiles are self-assembling macromolecular structures composed of amphiphiles where some biological functionalities are embedded. The introduction of biological motifs such as simple peptides, carbohydrates, or steroids into the structures of amphiphiles allows mimicking specific functions of biosystems thus representing a formidable approach leading to wide range of potential applications in drug delivery and biotechnology in general. One of the best examples of smart biofunctional amphiphiles is the peptide amphiphiles [49]. Peptide amphiphiles (PAs) are constructed by solid-phase peptide synthesis through the conjugation of a hydrophobic tail to a short peptide sequence. When dissolved in water, PAs tend to self-assemble into cylindrical aggregates of nanofibers (about $10 \mathrm{~nm}$ in width and microns in length), while the selection of the amino acids determines, through folding and hydrogen bonding, the generated (secondary, tertiary, and quaternary) structures.

The three main regions of PA (Figure 9) are, respectively, the hydrophobic tail forming the PA nanofiber core (region I), $\beta$-sheet forming amino acids that induce formation of a filamentous nanostructure with hydrogen bonds (central region II), and finally the (charged) hydrophilic moieties that facilitate PAs water solubility (region III) [49-51]. By changing amino acids types in region II the properties of PA assemblies can be tuned to accomplish specific functions, while bioactive sequences can be attached at the water-exposed surface of the peptide sequence. At specific ionic strengths it is possible to observe PAs gelation at low concentration $(<1 \mathrm{wt} . \%)$ with the formation of a fibrous mesh. In this case encapsulation and release of small drugs from PAs supramolecular gels can be regulated by the hydrophobic and ionic interactions between the drug and the PAs hydrogel. Release kinetics of PAs with the model drug Prodan bound at different sites in the PA nanostructure showed that release rate increases with decreasing packing density, decreasing order in the hydrophobic PA core, and decreasing $\beta$-sheet character in the peptide [51]. The study demonstrates the capacity to control drug release by exploiting supramolecular structure. Peptide materials are good candidates also for in vivo gelling for delivery of drugs and proteins as well as in regenerative medicine. In vivo gelation can be obtained by injecting the PA solution within preformed nanostructures at low ionic strength into the high ionic strength environment of biological tissues. During diffusion process into a biomembrane the drug travels from a dilute solution outside of the cell to a particular site in the cell. With the aim of elucidating kinetic mechanisms of molecular assembly and the main factors that ultimately influence the final morphology of nanostructures, coarse-grained molecular dynamics simulations have been performed on a system of 800 peptide amphiphiles (sequence palmitoyl- $\mathrm{Val}_{3} \mathrm{Ala}_{3} \mathrm{Glu}_{3}$ ) [52]. The study pointed out how 


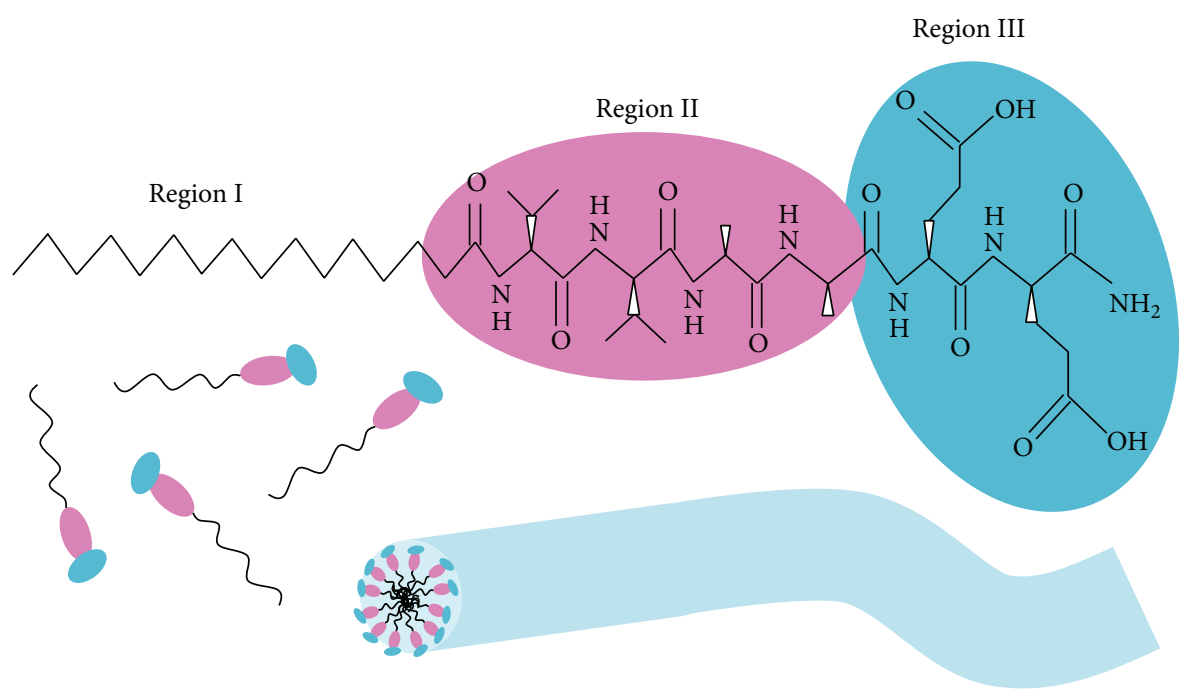

Figure 9: Chemical structure of peptide amphiphiles $E 2\left(\mathrm{C}_{16} \mathrm{~V}_{2} \mathrm{~A}_{2} \mathrm{E}_{2}\right)$ and their self-assembled nanofiber [49].

the self-assembly process occurs via a multistep process with transient intermediates including open networks of $\beta$-sheets, cylindrical nanofibers, and elongated micelles.

\section{Self-Assembly in Polymer-Based Amphiphiles: Linear, Hyperbranched, and Cross-Linked Morphologies}

Another important class of amphiphilic compounds are represented by macromolecular polymer-based amphiphiles. The concept of amphiphile can be extended, in fact, to polymeric amphiphile obtained by linking hydrophilic/hydrophobic polymer blocks through a covalent bond (amphiphilic block copolymers). Depending on the molecular topology and the binding sites in the building blocks, amphiphilic polymers can be classified into three main types: linear (block) copolymers, dendritic/star-shaped/hyperbranched polymers, and cross-linked polymers (or hydrogels) [53, 54].

8.1. Self-Assembly of Linear Block Copolymers. Self-assembly of linear block copolymers in selective solvents can be viewed in strong analogy to low molecular weight surfactant in water. On the other hand, since a block copolymer chain may consist of more than 400 monomeric units, the free energy per micelles is much larger for block copolymers than for traditional surfactants in water. This circumstance facilitates the entanglement between the polymer chains and stronger interblock interactions that prevent quick molecular exchange in comparison with traditional amphiphiles. Moreover, the possibility of molecular control by tuning the desired polymer composition and architecture makes these systems a versatile tool to study, in a convenient way, the rich and complex phenomenology in the field of colloidal science [53-57] and stimulates the route for the rational design and engineering of materials with desired properties [58, 59]. For example, Zeng et al. [60] recently provided a facile synthesis of peptide-RGD, poly(ethylene glycol), and cholesterol modified liposomes (RGD-PEG-Chol) with potential application as a drug delivery system of the poorly soluble antitumor drug paclitaxel (PTX). The drug carrier showed increased cellular uptake and sustained in vitro drug release behavior, while flow cytometry analysis revealed an increased cellular uptake of coumarin encapsulated in the RGD-PEG-Chol liposome for human umbilical vein endothelial cells (HUVEC). Moreover, the in vitro cytotoxicity shows that RGD-PTX liposome has significantly enhanced cytotoxicity of paclitaxel for melanoma cells [60]. Besides the linear AB diblock copolymers, most commonly thermoresponsive linear ABA triblock architecture obtained by versatile synthesis processes has attracted enormous interest and has already found broad application in biomedicine as tissue engineering and in drug/protein delivery $[61,62]$. Depending on the position of the hydrophobic block, ABA triblock copolymers in water form star-like (with central hydrophobic B block) or flowerlike micelles, with external hydrophobic A block forming a loop (i.e., a "petal"). In thermoresponsive ABA triblock copolymers, the temperature can be used as a trigger to form flower-type micelles or/and hydrogels at the higher concentrations. Recent studies on block polymeric micelles have demonstrated particular strength in solubilizing hydrophobic drugs in relevant doses without the inclusion of toxic organic solvents or surfactants $[63,64]$. Moreover, anticancer efficiency can be obtained by modifying the micelles surface with targeting ligands for specific recognition of receptors (overexpressed on the surface of tumor cells) [65]. While the hydrophobic block can be tailored to encapsulate drug molecules with a wide variety of structures, the hydrophilic block is generally formed of poly(ethylene glycol) (PEG), which assures the requested biocompatibility and the desired "stealth" characteristic that minimize unwanted interactions with cellular components. Polymers used in redox-responsive block copolymer systems often use poly(ethylene glycol) (PEG) in combination with biodegradable polymers such as 


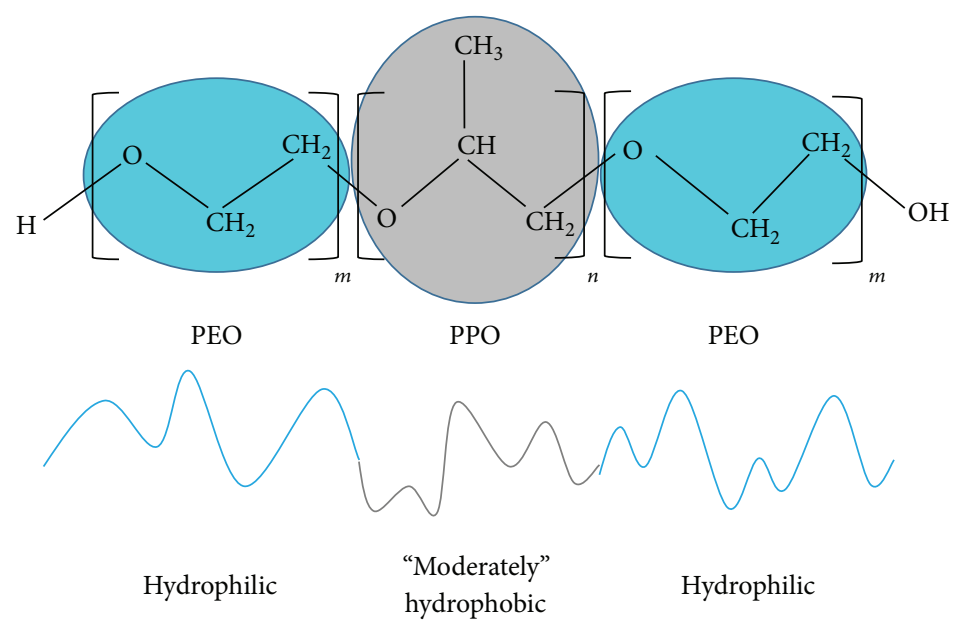

(a)

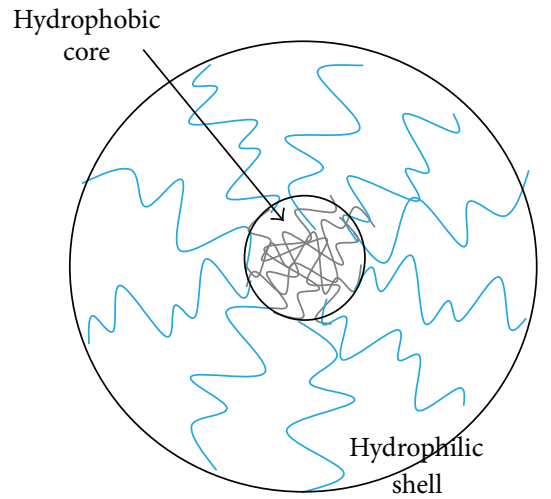

(b)

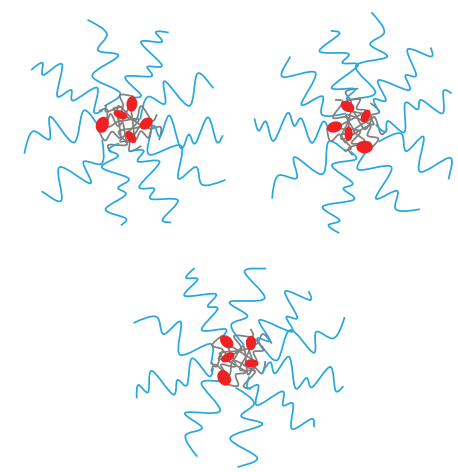

(c)

FIgURE 10: Chemical formula and structure of Pluronic-type $\mathrm{PEO}_{m}-\mathrm{PPO}_{n}-\mathrm{PEO}_{m}$ triblock copolymer (a). Core-shell micellar structure (b). Entrapment of drug in the micelles hydrophobic core (c).

polyesters poly(lactic acid), $\operatorname{poly}(\gamma$-methyl- $\varepsilon$-caprolactone $)$ [66].

A special class of ABA triblock copolymers are represented by the commercially available Pluronic-type class of amphiphilic poly(ethyleneoxide)-poly(propyleneoxide)poly(ethyleneoxide) $\mathrm{PEO}_{m}-\mathrm{PPO}_{n}-\mathrm{PEO}_{m}$ triblock copolymers (Figure 10). Apart from their widespread industrial applications in detergency, dispersion stabilization, foaming, emulsification, lubrication, and formulation of cosmetics, Pluronic copolymers represent a versatile component for applications in medicine, as drug delivery carriers, biological response modifiers, and pharmaceutical ingredients. A relevant number of studies involving Pluronic block copolymers as drug delivery systems or bioformulations for (pre)clinical use or trials are present in literature [67].

8.2. Hyperbranched, Star-Shaped, and Dendritic Polymers. Another interesting class of polymer-based amphiphiles are constructed by employing the highly branched three-dimensional polymer such as hyperbranched, star-shaped, and dendritic polymers. These classes of macromolecules have attracted a great attention owing to their interesting properties resulting from the (hyper)branched architecture and the high number of functional groups that generate poor molecular entanglement, high solubility, and generally low viscosity $[58,68,69]$. Dendrimers are highly monodispersed macromolecules with well-defined branched architecture consisting of dendritic cores and peripheral sites and represent fascinating building blocks for versatile application in host-guest, biotechnology, and catalytic chemistry [70, 71]. The (charged) surface end-groups strongly influence the interaction and the solubility as well as the molecular recognition ability, while the topological and chemical variety of the core region impart the requested size, absorption ability, and inclusion-release characteristics to the central cavity $[71,72]$. In comparison to dendrimers that request accurate multistep syntheses, hyperbranched polymers are usually synthesized in one-step processes and are considered to be ill-defined dendrimers due to their higher polydispersity. Recently, hyperbranched polymers have received more and more interest in both academic and industrial fields because of their advantages in facile one-pot synthesis, low cost, peculiar structures, excellent properties, and broad applications [73]. Applications in the field of nanoscience and technology highlighted in recent literature include catalysis, energy harvesting, photonics, drug delivery, and gene transfection [74, 75]. Within these supramolecular hyperbranched polymers 


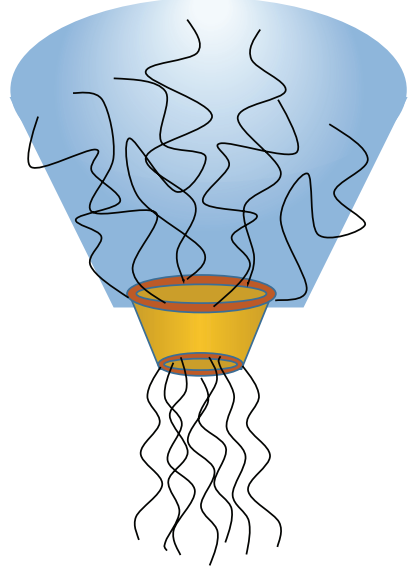

(a)

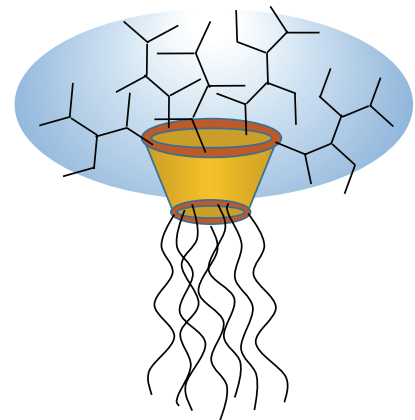

(b)

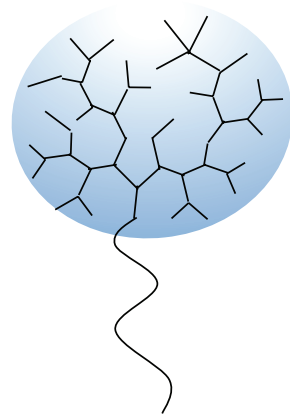

(c)

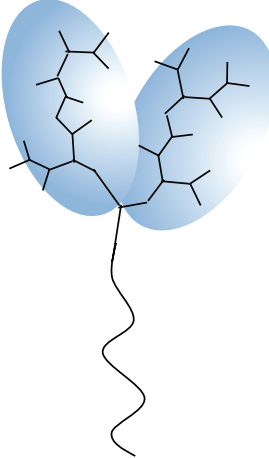

(d)

FIGURE 11: Schematic representation of complex shape amphiphiles: cuttlefish (a) [76], jellyfish (b) [77], and linear-dendrimer (c), linearhyperbranched (d) diblock copolymer.

linear-dendritic block copolymers and dendritic-dendritic block (or multiarm) copolymers appear as promising materials for different advanced applications such as controlled drug delivery, environmental-specific nanoreactors, artificial enzymes, and biosensors $[74,75]$. A schematic representation of some complex shape amphiphiles is reported in Figure 11.

Entanglement of multiple polymer segments by chemical synthesis has been employed to realize macromolecular amphiphiles with a large variety of different morphologies. For example, a series of novel fluorinated amphiphilic polymerization stabilizers of different architecture (diblock, grafted, or palm tree copolymers) were successfully prepared by reversible addition-fragmentation chain transfer controlled synthesis [78] while the synthesis and self-assembly processes of uniform aggregates of cuttlefish [76] and jellyfish [77] shaped modified cyclodextrin have been recently reported. It is worth mentioning that highly branched (co)polymers represent also fascinating and versatile building blocks for versatile construction of stimuli-responsive nanomaterials. In this respect photoresponsive supramolecular Janus hyperbranched polymer (JHBP) obtained on the basis of cyclodextrin/azobenzene host-guest molecular recognition selfassembly (and disassembly) processes [79] has been recently synthesized. The Janus hyperbranched polymer consisting of two distinct hyperbranched polymers (resembling a tree together with its root) was constructed by the noncovalent coupling between a hydrophobic hyperbranched poly(3ethyl-3-oxetanemethanol) with an apex of azobenzene (AZO) group and a hydrophilic hyperbranched polyglycerol with an apex of $\beta$-cyclodextrin (CD) group through the specific $A Z O / C D$ host-guest interactions. Simulations indicate that the material self-assembles into unilamellar bilayer vesicles with narrow size distribution, which disassembles reversibly under the UV light irradiation due to the trans-to-cis isomerization of the AZO groups. Moreover, TEM indicated that, under freeze-drying conditions, the vesicles further aggregate into colloidal crystal-like close-packed arrays [79].
8.3. Amphiphilic Polymer Conetworks (Hydrogels). Amphiphilic polymer conetworks consist of chemically (or physically) cross-linked polymer networks containing both hydrophilic (or polar) and hydrophobic monomers that can swell their volume in response to external stimuli or (internal) environment changes [80, 81]. These polymer networks are generally dispersed in water which form semisolid states (hydrogels). As represented in Figure 12(a) the main types of hydrogels are, respectively, the physical gel, formed by physical entanglement (without covalently linking) of polymer chains, the covalently linked networks (or cross-linked gels), and finally the interpenetrating networks consisting of networks formed cross-linking two (or more) different polymers. Due to its LCST (close to body temperature) and its fast on-off switching thermosensitive poly(N-isopropylacrylamide) (PNIPAAm) hydrogel with an interpenetrating polymer network (IPN) structure is one of the most intensely studied topics for biomedical applications [82]. When crosslinked into hydrogels, the coil-to-globule transition is followed by a decrease in the gel volume resulting in a fast diffusion controlled release of entrapped drug and solvent. Stimuli-responsive (smart) hydrogels can perform mechanical work in response to external changes of the physical properties of the system (stimuli) such as its temperature, electric field, and exposure to light [83]. Their applications include microfluidic and microactuators devices, superadsorbent, electrolyte batteries, and artificial muscles [83-85]. In biomedical research responsive hydrogel has found applications in biosensors, drug delivery, tissue engineering, and biomimetic materials [86-88].

The properties of hydrogels can be tuned to match the needs of specific applications by the choice of specific polymer (molecular structure and segments length) and the cross-linking mechanism and presence of acidic (or basic) polymer moieties, whose state of protonation can be controlled with $\mathrm{pH}$ or salt concentration. The $\mathrm{pH}$ sensitive gels have been mainly considered for the delivery 


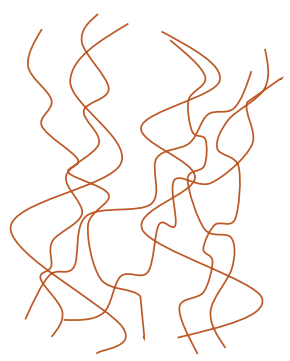

Physical gel

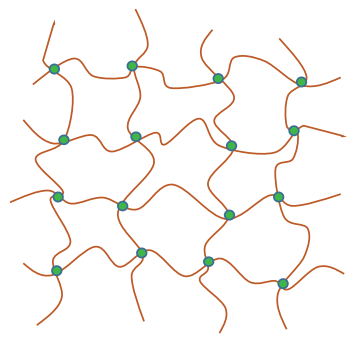

Covalently linked gel

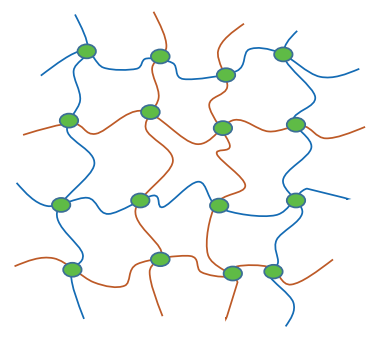

Interpenetrating network

(a)
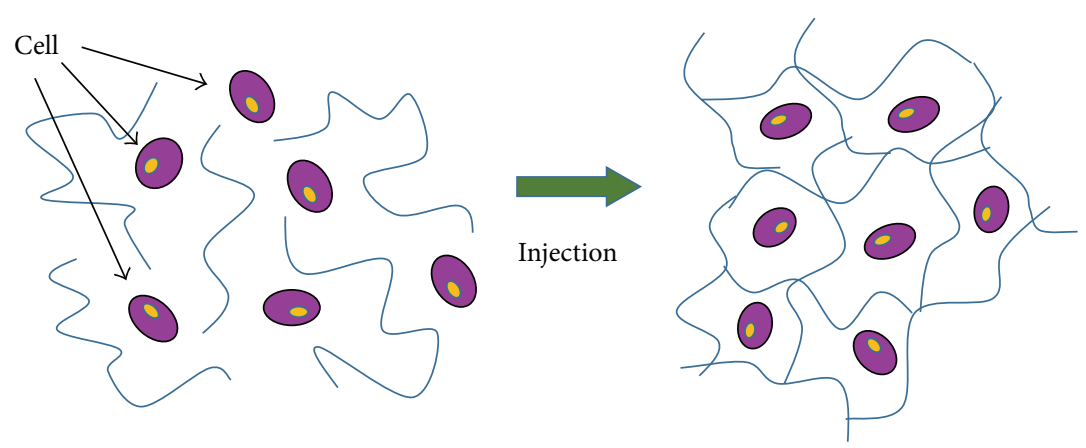

(b)

FIGURE 12: Schematic representation of the main types of hydrogels (a). In situ formation of a scaffold in tissue engineering, by injection into the wound of a polymer/cell solution and formation of a cross-linked polymer network (b).

of hydrophilic drugs, where the release of the molecules generally occurs by diffusion in the swollen polymer network. The $\mathrm{pH}$-responsive behavior can be useful to engineer devices for the targeted delivery of hydrophobic drugs. For example, the drug can be accurately localized within the hydrophobic pockets of the hydrogel (Figure 12(b)), while the hydrophobic drug's release can be externally controlled by varying the bath $\mathrm{pH}$ or ionic strength. Particularly interesting as drug delivery vehicles are the mesoordered hydrogels due to their high controllable porosity, large surface area, and pore size distribution. Formation of well-ordered mesoordered hydrogel particles (with $5 \mathrm{~nm}$ pore size) by means of cross-linking poly(ethylene glycol) diacrylate (PEG-DA) in the presence of surfactants has been recently reported [89]. Due to its molecular permeability and high mechanical strength in combination with a high water content $(>70 \%)$ interpenetrating network consisting of poly(ethylene glycol) and poly(acrylic acid) (PEG-PAA) has been developed and characterized for potential application as an artificial cornea or as replacement materials for the cartilage and other load-bearing anatomical structures [90]. In the presence of stereogenic center in the polymer, during the gel formation process chirality is transferred from singular molecules to nanoscale structures thus influencing morphology and global gelation behavior of hydrogels. Studies of hydrogel chirality have stimulated interdisciplinary research at the crossroad between material sciences, chemistry, and biomedicines with potential applications in microfluid devices, chiral recognition, enantiomeric separation, and controlled drug release $[83,86,91]$.

\section{Amphiphiles as Templates for the Synthesis of Mesoporous Nanostructures}

The peculiar structural characteristic of the amphiphile, specifically the simultaneous presence of polar and apolar moieties within the molecular architecture, allows the formation of closed structures (reversed micelles) which have been exploited successfully already in the 1980s for the synthesis of nanoparticles [92]. Thinking of such aggregates not in static sense, the templating effect can be traced back to their peculiar dynamical processes (with lifetimes of the order of milliseconds), while the intermicellar material exchange process, occurring on the same time scale, allows hydrophilic reactants to come rapidly in contact and react [93]. Moreover, the closed structure of the oriented monolayer of amphiphiles and their dispersion in a nonpolar environment could inhibit the unlimited growth of nanoparticles. In this respect the nanoparticles-amphiphiles soft interactions are responsible for nanoparticle coating, protection, and stabilization processes [94], while both size and shape of such templating structures can be modulated by opportunely changing the composition and the amphiphile nature.

The use of amphiphiles self-assembly has been demonstrated in promising bottom-up methods especially for designing alternative protocols for the self-assembly mechanism of supramolecular organic-inorganic nanostructures based on porous materials in which an amphiphilic macromolecular template drives the formation of nanostructures with peculiar final properties $[95,96]$. In this respect, combination of molecular interactions together with the ability to control 


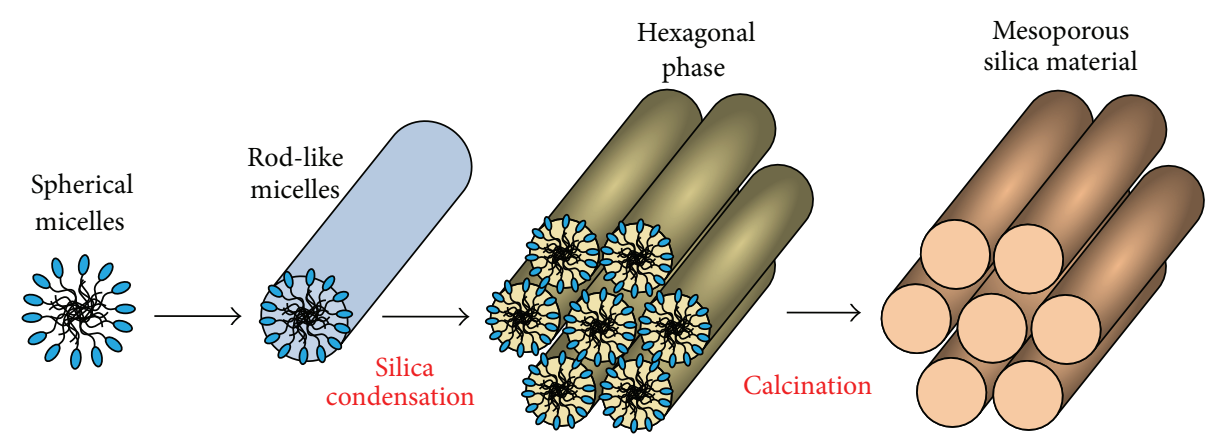

FIGURE 13: Formation mechanism of mesoporous silica materials. When the silica precursor is added to the amphiphiles solution the spherical micelles become elongated and arrange themselves in a hexagonal pattern while the silica walls are built. After the removal of surfactants by calcination only the mesoporous silica porous structure remains [104].

both length scale and structural morphologies [97-100] makes amphiphiles particularly interesting templates in the synthesis of porous materials with new characteristics and properties. Amphiphilic micelles templated mesoporous silica materials have attracted considerable interest in a wide range of applications including (heterogeneous) catalysis, adsorption and separation processes, electronics, optics, host-guest chemistry, and biotechnology [96, 101]. First synthesis of silica mesoporous nanoparticles with nonionic triblock polymers as template was reported by Zhao et al. in 1998 $[102,103]$. These materials were named SBA-X (Santa Barbara Amorphous), where $\mathrm{X}$ is a number corresponding to a specific pore structure and surfactant. For example, SBA-15, the most extensively studied mesoporous silica, has hexagonally ordered cylindrical pores synthesized using Pluronic P123 as a surfactant template.

The different stages of structure formation and driving forces controlling the synthesis of SBA-15 (sol-gel synthesis) are still only in part understood. The colloidal solution system (sol) is composed of the surfactant micelles dispersed in an aqueous solution in presence of a silica source (precursor) such as the organosilicate compound tetramethyl orthosilicate (TMOS) or tetraethyl orthosilicate (TEOS) or other alkoxides with longer alkyl chains. When the silica precursor is added to the sol it adsorbs on the micelles and hydrolyses while a silica network is gradually formed (a gel) in which the liquid is enclosed. Finally, the gel is heated (calcinated) and the surfactant is decomposed or oxidized under oxygen or air atmospheres and evaporates, while the porous silica network remains. The whole process is also referred to as soft templating, for the use of an organic material as (soft) template, or "cooperative self-assembly" as the amphiphile and the silica precursor assembly are simultaneous. In a variation of this method a much more concentrated (amphiphile) liquid crystalline phase solution is preformed before condensation of the inorganic framework, while the precursors are added in successive period of the synthesis process (liquid crystal templating). In Figure 13 a schematic representation of the formation mechanism of mesoporous silica materials is reported [104]. Investigation of the SBA-15 synthesis with SAXS, NMR, and TEM techniques indicated the adsorption of silica source on the hydrophilic PEO chains of the micelles during the hydrolysis [105]. The polymerization of the silica introduces an attractive interaction between the micelles which elongates in thread-like (cylindrical) micelles leading to the final formation of flocs of micelles. SAXS/SANS in situ experiment has also been employed to investigate the different phases of the self-assembly in solution of different 2D-hexagonal silica SBA-15 nanostructured materials [106] while the fine-tuning of the intrawall porosity and (primary) mesoporosity of SBA-15 has been obtained by addition of salt or by changing the temperature during the synthesis steps, as demonstrated recently by means of nitrogen sorption and small-angle X-ray diffraction experiments [107]. Many examples of soft templating use also ionic amphiphiles [108]. For example, a time-resolved synchrotron SAXS study investigated the synthesis of MCM-41 materials starting from a self-assembly process involving a Gemini (C16-416) surfactant as template [109]. Moreover the time-resolved, contrast-matched SANS investigation of Hollamby et al. [110] has shown the formation mechanisms of mesoporous silica nanoparticles (MSNs) in presence of CTAB amphiphiles.

With the aim of resolving the stability problem of synthesized zeolite-based porous nanostructures in solution the successful formation of a mesoporous spherical complex, driven by the incorporation of aluminosilicate in a dendrimer charged surface, has been recently demonstrated [111] by means of dynamic light scattering (DLS) and small-angle $\mathrm{X}$-ray scattering (SAXS) investigations. The use of highly branched macromolecular structure as template furnishes an enhancement of template stability in solution during the zeolite synthesis. In Figure 14 an example of the self-assembly of LTA zeolite synthesis in presence of PDMS-PEO block copolymer template is presented [100]. Recently Xu et al. reported an approach for designing a single (quaternary ammonium) head amphiphilic template with strong ordered self-assembling ability by employing the $\pi-\pi$ stacking interactions [112]. The noncovalent (aromatic-aromatic) $\pi-\pi$ stacking interactions have been shown to stabilize the micelle structure by introducing strong mutual interactions in the hydrophobic chain to block crystal growth by forming an extremely stable hydrophobic layer and giving an ordered orientation to the new designed MFI zeolitic (nanosheet) framework. In conclusion, it is worth mentioning that mesoporous 


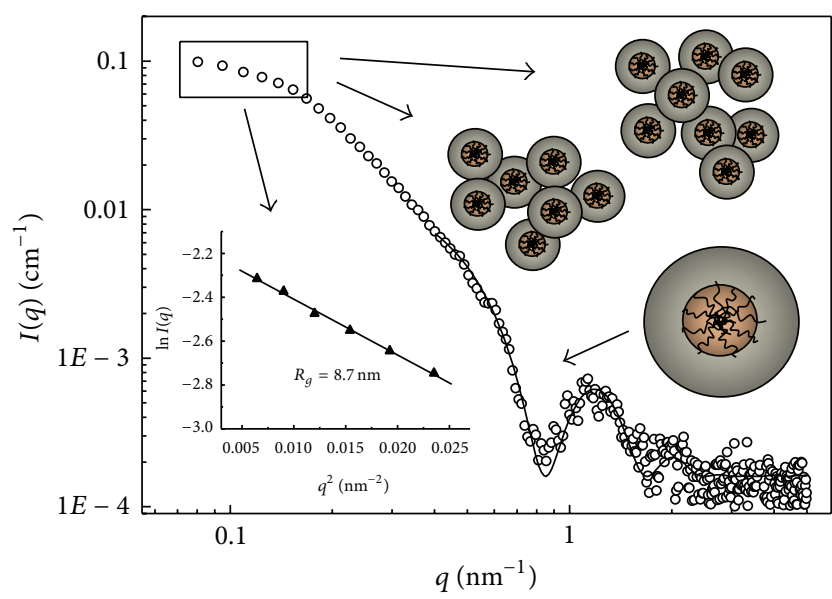

FIgURE 14: Small-angle X-ray scattering (SAXS) spectra during the LTA zeolite synthesis in presence of PDMS-PEO block copolymer as template. Core-shell structural approach (for $q>0.3 \mathrm{~nm}^{-1}$ ) and Guinier type SAXS analysis of aggregation subunits (for $q<$ $0.15 \mathrm{~nm}^{-1}$, inset).

silica nanoparticles (MSNs) are a highly promising platform for intracellular controlled release of drugs and biomolecules. Although their application in the field of intracellular drug delivery is still at the beginning very exciting breakthroughs have been achieved in the last years. The ability to functionalize the surface of the MSNs with organic groups was used as a way to incorporate functional molecules that are able to interact with intracellular structures and could then represent a promising vehicle for drug targeting applications [113-116].

\section{Hierarchical Self-Assembly and Supramolecular Amphiphiles}

In a traditional amphiphile the hydrophobic and hydrophilic parts are irreversibly connected together by covalent bonds. On the other hand a hierarchical self-assembly based on the concept of supramolecular amphiphiles can be realized by employing suitable noncovalent driving forces including hydrogen bonding, host-guest recognition, electrostatic forces, metal coordination, and $\pi-\pi$ stacking interaction. The reversible nature of noncovalent interactions allows a dynamic switching of structure, morphology, and function in response to various external stimuli, such as $\mathrm{pH}$, stress, temperature, and electromagnetic radiation [117-120]. For example, the self-assembly nanostructures could disassemble upon the activation of an external stimulus such as ultraviolet (UV) electromagnetic radiation or $\mathrm{pH}$, and this process can be exploited for potential applications in the field of controlled drug delivery. In this case, the effective final morphology is governed by the structural features of the component building block and by the balance between the hydrophobichydrophilic segments of the supramolecular amphiphiles $[121,122]$. For example, fully supramolecular polyrotaxanes (pseudopolyrotaxanes, PPR) consisting of $\alpha$-cyclodextrin rings and polyethylene glycol axes with end thymine groups have been synthesized for fluorescein (Fl) drug delivery system and used as biphase drug delivery systems [123]. Release of the secondary drug delivery systems from the primary drug delivery system was controlled by stimuli factors such as $\mathrm{pH}$. Due to the high versatility of the noncovalent supramolecular interactions between capping agents and pseudopolyrotaxanes, those components represent promising systems to deliver therapeutic agents such as anticancer drugs to target tissues.

One advantage of this strategy is the wide selection of mono- or multifunctionalized building blocks (e.g., monomer, hyperbranched polymer, and macrocycles) for supramolecular copolymers and a variety of synthetic methods using noncovalent interactions rather than chemical synthesis [121]. As reported in Figure 15, various noncovalent interactions can be adopted as driving forces to construct supramolecular amphiphiles.

Metal-organic coordination interaction can be employed in construction of complex self-assembled amphiphiles nanostructures. For example, a new type of Bola amphiphile bearing bipyrimidine (bpym-8) has been designed and synthesized by means of metal-ligand coordination interaction [124]. The generated structures are able to coordinate with metal ions and may provide a new approach in fabricating reversibly tunable supramolecular nanomaterials [125].

The $\pi-\pi$ stacking interactions, which involve direct attraction between aromatic rings (Figure 15(d)), have been implicated widely in supramolecular chemistry including DNA stabilization, drug intercalation, and protein structure and have great potential applications in biotechnology and material sciences. For example, supramolecular polymerization involving the $\pi-\pi$ stacking interaction of the hexa-peri-hexabenzocoronene $(\mathrm{HBC})$ molecules, the smallest fragment of graphene, has been employed in the self-assembly of hexabenzocoronene nanotubes [126]. Recently reduction-triggered breakable polymeric spherical micelles incorporated with methotrexate (MTX) were prepared using amphiphilic PAAg-PEG copolymers [127]. While, in normal conditions, most of the incorporated MTX in the micelles core was stabilized through the $\pi-\pi$ stacking with the phenyl groups in the backbone of the copolymers, in reductive environments (mimicking the intracellular compartments) the entire MTX payload could be quickly released due to the reductiontriggered breakage of the micelles. These micelles showed good antiproliferative activity against several cancer cell lines, including KB, 4T-1, and HepG2.

As previously stated electrostatic interaction plays a crucial role in the self-assembly process involved in ionic amphiphiles. On the other hand, in supramolecular approaches the electrostatic force is mainly involved in entanglement processes of opposite charge functionalities at the surface of nanoparticles. Recently a novel heterotopic nanoparticle formed exploiting the electrostatic interaction between anionic porphyrins TPPS entangled in cationic amphiphilic cyclodextrins indicates the important role of charge and size of the nanocarriers in the modulation of colloidal stability in solution [76]. More specifically the investigated porphyrin/ $\mathrm{CD}$ nanoaggregates, which are able to photosensitize singlet 


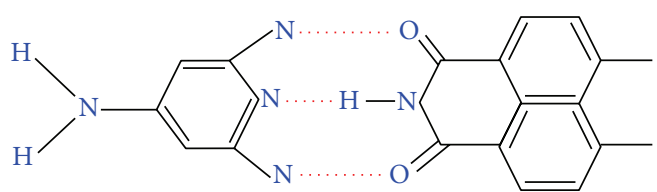

(a)
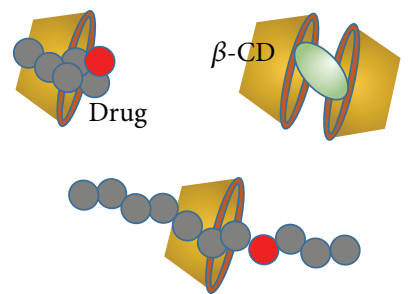

(b)

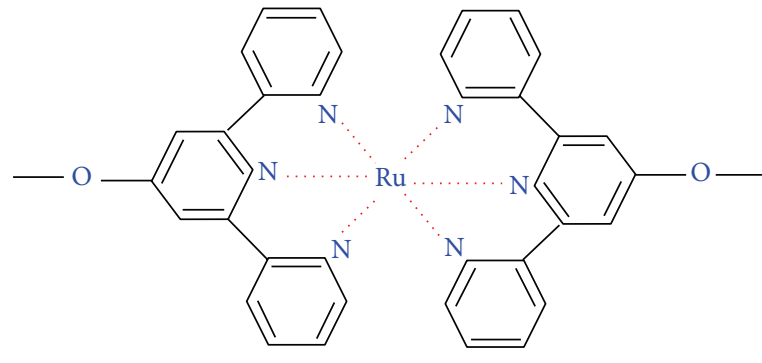

(c)

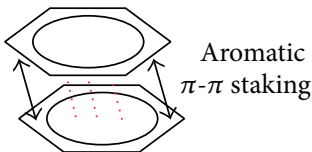

(d)

FIGURE 15: Noncovalent interactions involved in the construction of supramolecular amphiphiles: (a) multiple hydrogen bonding, (b) hostguest interaction based on $\beta$-cyclodextrin, (c) metal coordination, and (d) $\pi$ - $\pi$ stacking interaction.

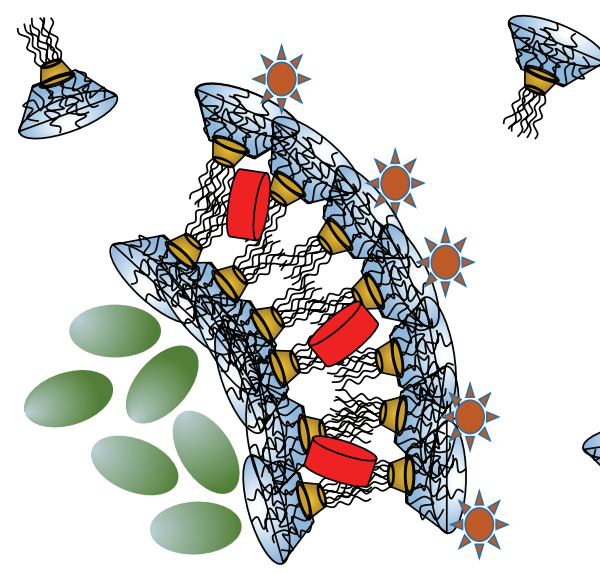

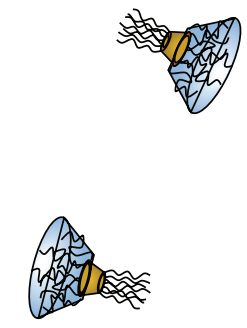

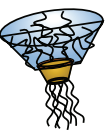

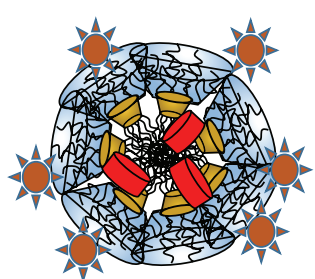

(b)

(a)
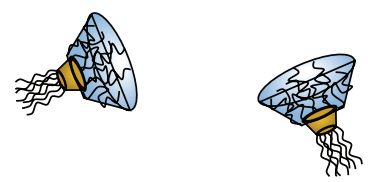

FIGURE 16: Ionic and nonionic drugs interacting with ionic amphiphilic cyclodextrins. Heterotopic vesicles (a) and micelles (b) nanoparticle formation during self-assembly.

oxygen production, appeared as an efficient system for delivering TPPS porphyrins into tumor cells and may be of potential interest for the development of agents for photodynamic therapy of tumors. On the basis of terminal group electrostatic interactions recently a supramolecular amphiphile has been constructed between the terminal carboxyl groups $(-\mathrm{COOH})$ of hydrophobic polylactic acid (PLA) and amino groups $\left(-\mathrm{NH}_{2}\right)$ of modified CDs [128]. Due to the biocompatibility of the building blocks the generated micellar structures in aqueous solution have been further explored as nanocarrier of anticancer drugs. It is worth pointing out that in most of the cases where the supramolecular self-assembly processes are involving electrostatic forces the cooperative charge effects may sensitively contribute with an enhancement of the stability in solution to the generated heterotopic colloid. In Figure 16 an illustrative example of possible heterotopic nanoparticle formation from hydrophobic and electrostatic interactions between ionic and nonionic drugs entangled in ionic amphiphilic cyclodextrins is presented [76].

Among the self-assembled hierarchical amphiphiles the construction of supramolecular nanostructures based on host-guest interaction provides a flexible platform for the development of smart nanomaterials and functional supramolecular devices. Molecular recognition of a host-guest system consists of a receptor molecule (host) interacting with a ligand molecule (guest) through noncovalent interactions [121, 129]. Among the variety of synthetic organic macromolecules used as molecular receptors to construct supramolecular amphiphiles distinctive properties have been obtained by 
introducing macrocyclic hosts into the supramolecular systems such as cyclodextrins [76], crown ethers [130], cucurbiturils [131], and calixarenes [132]. Cyclodextrins (CDs) have been extensively employed as highly performant receptors for molecular recognition as well as versatile building blocks to construct nanostructured functional materials. CDs have attracted the researcher interest for their ability to establish noncovalent interactions with a large variety of guest molecules that can fit into their cavity and form of inclusion complexes. The major driving forces of the formation of CD inclusion compounds are hydrophobic and van der Waals interactions between the inner surface of the CD ring and the hydrophobic sites on the guest. Based on the host-guest interaction between $\beta$-CD and azobenzene moieties recently a star-like polymer $\beta$-cyclodextrin-poly(l-lactide) and a linear polymer, azobenzene-poly(ethylene glycol), could selfassemble into a supramolecular amphiphilic copolymer $(\beta$ CD-PLLA@Azo-PEG) [129]. By tuning the ratio between the hydrophilic/hydrophobic chains the corresponding linearstar copolymer (supramolecular amphiphiles) further selfassembled into a wide range of morphologies including (sphere-like/carambola-like/naan-like) micelles, shuttle-like lamellae, tube-like fiber, and random curled-up lamellae.

Combination of the dynamic/reversible nature of noncovalent interactions with the new topological features and multifunctionality of building blocks provides a versatile strategy for preparation of nanostructured functional soft materials. This respect is of paramount importance and results in the ability to undergo dynamic switching of structure, morphology, and functions in response to various external stimuli.

Stimuli-responsive supramolecular amphiphiles self-assemblies have potential applications in a wide range of fields, including memory storage, smart supramolecular polymers nanodevices, and drug delivery systems [133, 134]. Among numerous external stimuli, electromagnetic radiation (light) is of special interest. For example, azobenzene, which has two isomers (trans and cis), can be recognized by $\beta-\mathrm{CD}$ reversibly upon light irradiation [134]. Due to its photoinduced E/Z-isomerization, azobenzene has been widely proved to be especially advantageous for inducing large structural (and molecular properties) changes in supramolecular systems. Another interesting aspect regards the manipulation of the aggregation/deaggregation processes by amphiphiles. Recently the fluorescence modulation of tetraphenylethylene and silole fluorophores by ionic amphiphiles has been utilized to modulate the aggregation of aggregation-induced emission (AIE) fluorophores in aqueous solutions and thus to tune their fluorescence intensities [135]. This approach may enable novel applications in chemosensors or biosensors based on aggregation/deaggregation and inhibition of internal rotations of AIE fluorophores (as tuned by ionic amphiphiles). This study can stimulate also the construction of new assays for the detection of certain biomolecules and new agents for targeted bioimaging. Macrocycles also show ability to regulate the surface activity amphiphiles. The macrocycle cucurbit[7]uril $(\mathrm{CB}[7])$ has been employed for tuning, in a controllable and reversible fashion, the surface activity (amphiphilicity) of Gemini surfactant by exploiting the host-guest interaction and the hindering effect of $\mathrm{CB}[7]$ [131]. In that case a sensitive variation of surface tension, foamability, and wettability has been obtained by preventing the two alkyl chains of the Gemini amphiphile from getting close to each other by bounding each of them with $\mathrm{CB}$ [7] macrocycle. Moreover, with the addition of the 1-adamantanamine hydrochloride, a competitive guest molecule having a stronger binding ability with $\mathrm{CB}$ [7], the two alkyl chains are expelled from the cavity of $\mathrm{CB}$ [7] and get closed again, with a consequent recovery of the surface activity. In conclusion, it is worth pointing out how the synchronization of multiple interactions in a synergistic way yields to higher-order supramolecular architecture in a manner similar to biological systems. For example, a series of diamond-like supramolecular frameworks with mesoporous cavities has been recently assembled into the hierarchical complex structures, starting from metal-imidazolate coordination cages and various anions. In these cases multiple weak interactions, such as covalent bonds, dative bonds, and $\mathrm{CH}-\mathrm{X}$ hydrogen bonds (where $\mathrm{X}=\mathrm{O}, \mathrm{F}$, and $\pi$ ), act as a supportive interaction to direct the cooperative self-assembly of large coordination cages into hierarchical mesoporous architecture with high aspect ratio [136]. The generated crystalline supramolecular framework of mesoporous cavities is able to trap organic dye (or bioactive) molecules and specific drugs. The employment of such self-assembly strategies has recently provided a powerful platform for the construction of advanced materials from large aggregates of ordered mesoporous supramolecular structures [137-139].

\section{Conclusion}

The investigation of the amphiphiles self-assembly can be considered a precursor of the development of the bottomup approaches in modern nanotechnology. In this tutorial review we provide a fundamental description of amphiphile assembly, followed by an application-oriented description of advanced assembly of amphiphilic nanostructures. We point out how effective segregation of hydrophilic and hydrophobic regions represents the primary noncovalent driving force for the formation of structures such as micelles, vesicles, bilayers, microemulsions, and lyotropic liquid crystals. Design of more complex structures can be obtained by introducing structure directing interactions that influence, in a fascinating way, the morphology of the final assemblies. Moreover the incorporation of functional motives such as simple peptides or carbohydrates has enormously increased during the last few years, leading to the design of dedicated bioinspired amphiphiles. In this respect a large variety of synthetic systems are able to self-assemble in a highly ordered fashion capable of mimicking biological activity thus playing an important role in a variety of applications including drug release systems, tissue engineering, and gene therapy. Selfassembly of amphiphilic molecules, which has been the subject of intense research activity for colloid, polymer, and materials science for several years, continues to attract the renewed interest of researchers involved in nanoscience, combining simple processes of assembly with the more advanced concept of supramolecular chemistry which is at the heart of modern bottom-up nanotechnology. 


\section{Conflict of Interests}

The authors declare that there is no conflict of interests regarding the publication of this paper.

\section{Acknowledgments}

Part of the experimental results has been performed with the financial support of dedicated programs for the access to Synchrotron Radiation Facility (ESRF (Grenoble), ElettraTrieste, and Soleil (Saint Aubin)). The work of Mikhail A. Kiselev was supported by the Russian Scientific Foundation (Project no. 14-12-00516). Domenico Lombardo acknowledges funding from Marie Curie Actions under EU FP7 Initial Training Network SNAL 608184.

\section{References}

[1] J. N. Israelachvili, "Thermodynamic and geometric aspects of amphiphile aggregation into micelles, vesicles and bilayers, and the interactions between them," in Physics of Amphiphiles: Micelles, Vesicles and Microemulsions, V. Degiorgio and M. Corti, Eds., pp. 24-58, North-Holland, Amsterdam, The Netherlands, 1985.

[2] C. Domb, J. L. Lebowitz, G. Gompper, and M. Schick, SelfAssembling Amphiphilic Systems, Phase Transitions and Critical Phenomena, Academic Press, London, UK, 1994.

[3] S. C. Glotzer and M. J. Solomon, "Anisotropy of building blocks and their assembly into complex structures," Nature Materials, vol. 6, no. 8, pp. 557-562, 2007.

[4] J. Katsaras and T. Gutberlet, Lipid Bilayers Structure and Interactions, Springer, Berlin, Germany, 2001.

[5] J. M. Seddon and R. H. Templer, "Polymorphism of lipid-water systems," in Handbook of Biological Physics, R. Lipowsky and E. Sackmann, Eds., vol. 1, chapter 3, pp. 97-160, Elsevier Science Publisher BV, 1995.

[6] K. Holmberg, B. Jonsson, B. Kronberg, and B. Lindman, Surfactants and Polymers in Aqueous Solution, John Wiley \& Sons, Chichester, UK, 2nd edition, 2002.

[7] M. J. Rosen, Surfactants and Interfacial Phenomena, Wiley, New York, NY, USA, 2nd edition, 1989.

[8] J. N. Israelachvili, Intermolecular and Surface Forces, Academic Press, New York, NY, USA, 2nd edition, 1992.

[9] X. Cui, S. Mao, M. Liu, H. Yuan, and Y. Du, "Mechanism of surfactant micelle formation," Langmuir, vol. 24, no. 19, pp. 10771-10775, 2008.

[10] C. A. Angell, P. H. Poole, and J. Shao, "Glass-forming liquids, anomalous liquids, and polyamorphism in liquids and biopolymers," Il Nuovo Cimento D, vol. 16, no. 8, pp. 993-1025, 1994.

[11] P. G. Debenedetti and F. H. Stillinger, "Supercooled liquids and the glass transition," Nature, vol. 410, no. 6825, pp. 259-267, 2001.

[12] S. Magazù, F. Migliardo, and A. Benedetto, "Puzzle of protein dynamical transition," Journal of Physical Chemistry B, vol. 115, no. 24, pp. 7736-7743, 2011.

[13] B. Varga, F. Migliardo, E. Takacs, B. Vertessy, S. Magazù, and M. T. F. Telling, "Study of solvent-protein coupling effects by neutron scattering," Journal of Biological Physics, vol. 36, no. 2, pp. 207-220, 2010.

[14] C. Tanford, The Hydrophobic Effect: Formation of Micelles and Biological Membranes, Krieger, Malabar, Fla, USA, 1991.
[15] J. L. Finney, "The structural basis of the hydrophobic interaction," in Hydration Processes in Biology, M.-C. Bellissent-Funel, Ed., pp. 115-124, IOS Press, 1999.

[16] D. T. Bowron, A. Filipponi, M. A. Roberts, and J. L. Finney, "Hydrophobic hydration and the formation of a clathrate hydrate," Physical Review Letters, vol. 81, no. 19, pp. 4164-4167, 1998.

[17] S. Magazù, F. Migliardo, and M. T. F. Telling, "Study of the dynamical properties of water in disaccharide solutions," European Biophysics Journal, vol. 36, no. 2, pp. 163-171, 2007.

[18] P. W. Fenimore, H. Frauenfelder, S. Magazù et al., "Concepts and problems in protein dynamics," Chemical Physics, vol. 424, pp. 2-6, 2013.

[19] E. J. W. Verwey and J. T. G. Overbeek, Theory of the Stability of Lyophobic Colloids, Elsevier, Amsterdam, The Netherlands, 1948.

[20] R. J. Hunter, Foundations of Colloid Science, vol. 1-2, Oxford University Press, New York, NY, USA, 1986.

[21] J. P. Hansen and I. A. McDonald, Theory of Simple Liquids, Academic Press, New York, NY, USA, 1986.

[22] L. Belloni, "Colloidal interactions," Journal of Physics Condensed Matter, vol. 12, no. 46, pp. R549-R587, 2000.

[23] D. D. Lasic, "Applications of liposomes," in Handbook of Biological Physics, R. Lipowsky and E. Sackmann, Eds., vol. 1, pp. 491-519, Elsevier, Amsterdam, The Netherlands, 1995.

[24] A. Gezae Daful, V. A. Baulin, J. Bonet Avalos, and A. D. MacKie, "Accurate critical micelle concentrations from a microscopic surfactant model," Journal of Physical Chemistry B, vol. 115, no. 13, pp. 3434-3443, 2011.

[25] H. Zettl, Y. Portnoy, M. Gottlieb, and G. Krausch, "Investigation of micelle formation by fluorescence correlation spectroscopy," Journal of Physical Chemistry B, vol. 109, no. 27, pp. 13397-13401, 2005.

[26] M. Méndez-Pérez, B. Vaz, L. García-Río, and M. Pérez-Lorenzo, "Polymeric premicelles as efficient lipophilic nanocarriers: extending drug uptake to the submicellar regime," Langmuir, vol. 29, no. 36, pp. 11251-11259, 2013.

[27] R. Hadgiivanova and H. Diamant, "Premicellar aggregation of amphiphilic molecules: aggregate lifetime and polydispersity," Journal of Chemical Physics, vol. 130, Article ID 114901, 2009.

[28] V. A. Frolov, A. V. Shnyrova, and J. Zimmerberg, "Lipid polymorphisms and membrane shape," Cold Spring Harbor Perspectives in Biology, vol. 3, no. 11, Article ID a004747, 2011.

[29] R. A. Karjiban, N. S. Shaari, U. V. Gunasakaran, and M. Basri, "A coarse-grained molecular dynamics study of DLPC, DMPC, DPPC, and DSPC mixtures in aqueous solution," Journal of Chemistry, vol. 2013, Article ID 931051, 6 pages, 2013.

[30] M. A. Kiselev, P. Lesieur, A. M. Kisselev et al., "A sucrose solutions application to the study of model biological membranes," Nuclear Instruments and Methods in Physics Research, Section A: Accelerators, Spectrometers, Detectors and Associated Equipment, vol. 470, no. 1-2, pp. 409-416, 2001.

[31] M. Kotlarchyk, S.-H. Chen, J. S. Huang, and M. W. Kim, "Structure of three-component microemulsions in the critical region determined by small-angle neutron scattering," Physical Review A, vol. 29, article 2054, 1984.

[32] S. H. Chen, D. Lombardo, F. Mallamace, N. Micali, S. Trusso, and C. Vasi, "Small-angle light scattering in microemulsions (spinodal decomposition)," in Trends in Colloid and Interface Science VII, vol. 93 of Progress in Colloid \& Polymer Science, pp. 311-316, Steinkopff, 1993. 
[33] P. Schurtenberger, "Static and dynamic properties of micelles, microemulsions and vesicles," Current Opinion in Colloid \& Interface Science, vol. 1, no. 6, pp. 773-778, 1996.

[34] S. Buček, S. Kralj, and T. J. Sluckin, "Hysteresis in twodimensional liquid crystal models," Advances in Condensed Matter Physics, vol. 2015, Article ID 834867, 10 pages, 2015.

[35] D. Demus, J. Goodby, G. W. Gray, H. W. Spiess, and V. Vill, Handbook of Liquid Crystals, Wiley, Weinheim, Germany, 1998.

[36] Y. Yusuf, S. Yamaguchi, S. Kawano et al., "Polar liquid crystal elastomers cross linked far from thermodynamic phase transitions: dislocation loops in smectic clusters," Advances in Condensed Matter Physics, vol. 2013, Article ID 752060, 11 pages, 2013.

[37] R. Zana, Dynamics of Surfactant Self-Assemblies: Micelles, Microemulsions, Vesicles and Lyotropic Phases, edited by R. Zana, Taylor \& Francis, London, UK, 2005.

[38] C. Fong, T. Le, and C. J. Drummond, "Lyotropic liquid crystal engineering-ordered nanostructured small molecule amphiphile self-assembly materials by design," Chemical Society Reviews, vol. 41, no. 3, pp. 1297-1322, 2012.

[39] P. Calandra, V. Turco Liveri, A. M. Ruggirello, M. Licciardi, D. Lombardo, and A. Mandanici, "Anti-Arrhenian behaviour of conductivity in octanoic acid-bis(2-ethylhexyl)amine systems: a physico-chemical study," Journal of Materials Chemistry C, vol. 3, no. 13, pp. 3198-3210, 2015.

[40] V. De Giorgio and M. Corti, Eds., Physics of Amphiphiles: Micelles, Vesicles and Microemulsions, North-Holland, 1985.

[41] M. Yamada and I. Honma, "Anhydrous protonic conductivity of a self-assembled acid-base composite material," Journal of Physical Chemistry B, vol. 108, no. 18, pp. 5522-5526, 2004.

[42] P. Calandra, I. Nicotera, C. O. Rossi, and V. T. Liveri, "Dynamical properties of self-assembled surfactant-based mixtures: triggering of one-dimensional anomalous diffusion in bis(2ethylhexyl)phosphoric acid/n-octylamine systems," Langmuir, vol. 29, no. 48, pp. 14848-14854, 2013.

[43] T. A. Camesano and R. Nagarajan, "Micelle formation and CMC of gemini surfactants: a thermodynamic model," Colloids and Surfaces A: Physicochemical and Engineering Aspects, vol. 167, no. 1-2, pp. 165-177, 2000.

[44] J.-H. Fuhrhop and T. Wang, "Bolaamphiphiles," Chemical Reviews, vol. 104, no. 6, pp. 2901-2937, 2004.

[45] F.-Y. Zhai, W. Huang, G. Wu et al., "Nanofibers with very fine core-shell morphology from anisotropic micelle of amphiphilic crystalline-coil block copolymer," ACS Nano, vol. 7, no. 6, pp. 4892-4901, 2013.

[46] R. L. B. Selinger, J. V. Selinger, A. P. Malanoski, and J. M. Schnur, "Shape selection in chiral self-assembly," Physical Review Letters, vol. 93, no. 15, Article ID 158103, 2004.

[47] I. A. Nyrkova and A. N. Semenov, "Twisted surfactant structures: an advanced theoretical model," Soft Matter, vol. 6, no. 3, pp. 501-516, 2010.

[48] Z. Wu, Y. Yan, and J. Huang, "Advanced molecular self-assemblies facilitated by simple molecules," Langmuir, vol. 30, no. 48 , pp. 14375-14384, 2014.

[49] J. D. Hartgerink, E. Beniash, and S. I. Stupp, "Self-assembly and mineralization of peptide-amphiphile nanofibers," Science, vol. 294, no. 5547, pp. 1684-1688, 2001.

[50] P. A. Korevaar, C. J. Newcomb, E. W. Meijer, and S. I. Stupp, "Pathway selection in peptide amphiphile assembly," Journal of the American Chemical Society, vol. 136, no. 24, pp. 8540-8543, 2014.
[51] J. B. Matson, C. J. Newcomb, R. Bitton, and S. I. Stupp, "Nanostructure-templated control of drug release from peptide amphiphile nanofiber gels," Soft Matter, vol. 8, no. 13, pp. 3586$3595,2012$.

[52] I. W. Fu, C. B. Markegard, and H. D. Nguyen, "Solvent effects on kinetic mechanisms of self-assembly by peptide amphiphiles via molecular dynamics simulations," Langmuir, vol. 31, no. 1, pp. 315-324, 2015.

[53] P. Alexandridis and B. Lindman, Amphiphilic Block Copolymers: Self-Assembly and Applications, Elsevier, Amsterdam, The Netherlands, 2000.

[54] Y. Mai and A. Eisenberg, "Self-assembly of block copolymers," Chemical Society Reviews, vol. 41, no. 18, pp. 5969-5985, 2012.

[55] S. H. Chen, F. Mallamace, A. Faraone, P. Gambadauro, D. Lombardo, and W. R. Chen, "Observation of a re-entrant kinetic glass transition in a micellar system with temperaturedependent attractive interaction," European Physical Journal E, vol. 9, no. 3, pp. 283-286, 2002.

[56] P. Alexandridis, "Amphiphilic copolymers and their applications," Current Opinion in Colloid \& Interface Science, vol. 1, no. 4, pp. 490-501, 1996.

[57] S. Manet, A. Lecchi, M. Impéror-Clerc et al., "Structure of micelles of a nonionic block copolymer determined by SANS and SAXS," Journal of Physical Chemistry B, vol. 115, no. 39, pp. 11318-11329, 2011.

[58] R. Dong, Y. Zhou, X. Huang, X. Zhu, Y. Lu, and J. Shen, "Functional supramolecular polymers for biomedical applications," Advanced Materials, vol. 27, no. 3, pp. 498-526, 2015.

[59] C. Tan, Y. Wang, and W. Fan, "Exploring polymeric micelles for improved delivery of anticancer agents: recent developments in preclinical studies," Pharmaceutics, vol. 5, no. 1, pp. 201-219, 2013.

[60] S. Zeng, F. Wu, B. Li et al., "Synthesis, characterization, and evaluation of a novel amphiphilic polymer RGD-PEG-chol for target drug delivery system," The Scientific World Journal, vol. 2014, Article ID 546176, 10 pages, 2014.

[61] L. Zhang and A. Eisenberg, "Multiple morphologies of 'crew-cut' aggregates of polystyrene- $b$-poly(acrylic acid) block copolymers," Science, vol. 268, no. 5218, pp. 1728-1731, 1995.

[62] P. Alexandridis and T. A. Hatton, "Poly(ethylene oxide)poly(propylene oxide)-poly(ethylene oxide) block copolymer surfactants in aqueous solutions and at interfaces: thermodynamics, structure, dynamics, and modeling," Colloids and Surfaces A: Physicochemical and Engineering Aspects, vol. 96, no. 1-2, pp. 1-46, 1995.

[63] W. Zhao, D. Chen, Y. Hu, G. M. Grason, and T. P. Russell, "ABC triblock copolymer vesicles with mesh-like morphology," ACS Nano, vol. 5, no. 1, pp. 486-492, 2011.

[64] S. Toughraï, V. Malinova, R. Masciadri et al., "Reductionsensitive amphiphilic triblock copolymers self-assemble into stimuli-responsive micelles for drug delivery," Macromolecular Bioscience, vol. 15, no. 4, pp. 481-489, 2015.

[65] A. M. Butt, M. C. I. M. Amin, H. Katas, N. Sarisuta, W. Witoonsaridsilp, and R. Benjakul, "In vitro characterization of pluronic F127 and D- $\alpha$-tocopheryl polyethylene glycol 1000 succinate mixed micelles as nanocarriers for targeted anticancer-drug delivery," Journal of Nanomaterials, vol. 2012, Article ID 916573, 11 pages, 2012.

[66] H. L. Sun, B. N. Guo, R. Cheng, F. H. Meng, H. Y. Liu, and Z. Y. Zhong, "Biodegradable micelles with sheddable poly(ethylene glycol) shells for triggered intracellular release of doxorubicin," Biomaterials, vol. 30, no. 31, pp. 6358-6366, 2009. 
[67] A. Pitto-Barry and N. P. E. Barry, "Pluronic block-copolymers in medicine: from chemical and biological versatility to rationalisation and clinical advances," Polymer Chemistry, vol. 5, no. 10, pp. 3291-3297, 2014.

[68] F. Wurm and H. Frey, "Linear-dendritic block copolymers: the state of the art and exciting perspectives," Progress in Polymer Science, vol. 36, no. 1, pp. 1-52, 2011.

[69] R. Dong, Y. Zhou, and X. Zhu, "Supramolecular dendritic polymers: from synthesis to applications," Accounts of Chemical Research, vol. 47, no. 7, pp. 2006-2016, 2014.

[70] D. A. Tomalia, H. Baker, J. Dewald, M. Hall, G. Kallos, and S. Martin, "A new class of polymers: starburst-dendritic macromolecules," Polymer Journal, vol. 9, no. 1, pp. 117-132, 1985.

[71] S. C. Zimmerman and L. J. Lawless, Topics in Current Chemistry: Supramolecular Chemistry of Dendrimers, vol. 217, Springer, New York, NY, USA, 2001.

[72] D. Lombardo, "Modeling dendrimers charge interaction in solution: relevance in biosystems," Biochemistry Research International, vol. 2014, Article ID 837651, 10 pages, 2014.

[73] Y. Segawa, T. Higashihara, and M. Ueda, "Synthesis of hyperbranched polymers with controlled structure," Polymer Chemistry, vol. 4, no. 6, pp. 1746-1759, 2013.

[74] F. Sun, X. Luo, L. Kang, X. Peng, and C. Lu, "Synthesis of hyperbranched polymers and their applications in analytical chemistry," Polymer Chemistry, vol. 6, no. 8, pp. 1214-1225, 2015.

[75] D. Yan, C. Gao, and H. Frey, Eds., Hyperbranched Polymers Synthesis, Properties, and Applications, John Wiley \& Sons, Hoboken, NJ, USA, 2011.

[76] A. Mazzaglia, N. Angelini, D. Lombardo et al., "Amphiphilic cyclodextrin carriers embedding porphyrins: charge and size modulation of colloidal stability in heterotopic aggregates," Journal of Physical Chemistry B, vol. 109, no. 15, pp. 7258-7265, 2005.

[77] S. Shao, J. Si, J. Tang, M. Sui, and Y. Shen, "Jellyfish-shaped amphiphilic dendrimers: synthesis and formation of extremely uniform aggregates," Macromolecules, vol. 47, no. 3, pp. 916-921, 2014.

[78] D. Alaimo, A. Beigbeder, P. Dubois, G. Broze, C. Jérôme, and B. Grignard, "Block, random and palm-tree amphiphilic fluorinated copolymers: controlled synthesis, surface activity and use as dispersion polymerization stabilizers," Polymer Chemistry, vol. 5, no. 18, pp. 5273-5282, 2014.

[79] Y. Liu, C. Yu, H. Jin et al., "A supramolecular Janus hyperbranched polymer and its photoresponsive self-assembly of vesicles with narrow size distribution," Journal of the American Chemical Society, vol. 135, no. 12, pp. 4765-4770, 2013.

[80] G. Erdodi and J. P. Kennedy, "Amphiphilic conetworks: definition, synthesis, applications," Progress in Polymer Science, vol. 31, no. 1, pp. 1-18, 2006.

[81] M. M. E. Koenigs, A. Pal, H. Mortazavi, G. M. Pawar, C. Storm, and R. P. Sijbesma, "Tuning cross-link density in a physical hydrogel by supramolecular self-sorting," Macromolecules, vol. 47, no. 8, pp. 2712-2717, 2014.

[82] L.-W. Xia, R. Xie, X.-J. Ju, W. Wang, Q. Chen, and L.-Y. Chu, "Nano-structured smart hydrogels with rapid response and high elasticity," Nature Communications, vol. 4, article 2226, 2013.

[83] E. M. Ahmed, "Hydrogel: preparation, characterization, and applications: a review," Journal of Advanced Research, vol. 6, no. 2, pp. 105-121, 2015.
[84] D. J. Beebe, J. S. Moore, J. M. Bauer et al., "Functional hydrogel structures for autonomous flow control inside microfluidic channels," Nature, vol. 404, no. 6778, pp. 588-590, 2000.

[85] Y. Takashima, S. Hatanaka, M. Otsubo et al., "Expansioncontraction of photoresponsive artificial muscle regulated by host-guest interactions," Nature Communications, vol. 3, article 1270, 2012.

[86] D. Seliktar, "Designing cell-compatible hydrogels for biomedical applications," Science, vol. 336, no. 6085, pp. 1124-1128, 2012.

[87] R. Arora, G. Aggarwal, S. L. Harikumar, and K. Kaur, "Nanoemulsion based hydrogel for enhanced transdermal delivery of ketoprofen," Advances in Pharmaceutics, vol. 2014, Article ID 468456, 12 pages, 2014.

[88] P. Chen, X. Wang, Y. Dong, and X. Hu, "Development of a layerby-layer assembled film on hydrogel for ocular drug delivery," International Journal of Polymer Science, vol. 2015, Article ID 535092, 9 pages, 2015.

[89] M. Wallin, A. Altskär, L. Nordstierna, and M. Andersson, "Meso-ordered PEG-based particles," Langmuir, vol. 31, no. 1, pp. 13-16, 2015.

[90] D. Myung, W. U. Koh, J. M. Ko et al., "Biomimetic strain hardening in interpenetrating polymer network hydrogels," Polymer, vol. 48, no. 18, pp. 5376-5387, 2007.

[91] J.-S. Shen, G.-J. Mao, Y.-H. Zhou, Y.-B. Jiang, and H.-W. Zhang, "A ligand-chirality controlled supramolecular hydrogel," Dalton Transactions, vol. 39, no. 30, pp. 7054-7058, 2010.

[92] J. H. Fendler, "Atomic and molecular clusters in membrane mimetic chemistry," Chemical Reviews, vol. 87, no. 5, pp. 877899, 1987.

[93] P. D. I. Fletcher, A. M. Howe, and B. H. Robinson, “The kinetics of solubilisate exchange between water droplets of a water-inoil microemulsion," Journal of the Chemical Society, Faraday Transactions 1: Physical Chemistry in Condensed Phases, vol. 83, no. 4, pp. 985-1006, 1987.

[94] A. Longo, P. Calandra, M. P. Casaletto, C. Giordano, A. M. Venezia, and V. Turco Liveri, "Synthesis and physico-chemical characterization of gold nanoparticles softly coated by AOT," Materials Chemistry and Physics, vol. 96, no. 1, pp. 66-72, 2006.

[95] E. Prouzet and T. J. Pinnavaia, "Assembly of mesoporous molecular sieves containing wormhole motifs by a nonionic surfactant pathway: control of pore size by synthesis temperature," Angewandte Chemie-International Edition, vol. 36, no. 5, pp. 516-518, 1997.

[96] P. Botella, A. Corma, and M. Quesada, "Synthesis of ordered mesoporous silica templated with biocompatible surfactants and applications in controlled release of drugs," Journal of Materials Chemistry, vol. 22, no. 13, pp. 6394-6401, 2012.

[97] Y. Deng, J. Wei, Z. Sun, and D. Zhao, "Large-pore ordered mesoporous materials templated from non-Pluronic amphiphilic block copolymers," Chemical Society Reviews, vol. 42, no. 9, pp. 4054-4070, 2013.

[98] F. Mallamace, R. Beneduci, P. Gambadauro, D. Lombardo, and S. H. Chen, "Glass and percolation transitions in dense attractive micellar system," Physica A: Statistical Mechanics and Its Applications, vol. 302, no. 1-4, pp. 202-219, 2001.

[99] C. Yu, J. Fan, B. Tian, G. D. Stucky, and D. Zhao, "Synthesis of mesoporous silica from commercial poly(ethylene oxide)/poly(butylene oxide) copolymers: toward the rational design of ordered mesoporous materials," The Journal of Physical Chemistry B, vol. 107, no. 48, pp. 13368-13375, 2003. 
[100] L. Bonaccorsi, P. Calandra, M. A. Kiselev, H. Amenitsch, E. Proverbio, and D. Lombardo, "Self-assembly in poly(dimethylsiloxane)-poly(ethylene oxide) block copolymer template directed synthesis of linde type A zeolite," Langmuir, vol. 29, no. 23, pp. 7079-7086, 2013.

[101] X. Zhang, V. Thavasi, S. G. Mhaisalkar, and S. Ramakrishna, "Novel hollow mesoporous $1 \mathrm{D} \mathrm{TiO}_{2}$ nanofibers as photovoltaic and photocatalytic materials," Nanoscale, vol. 4, no. 5, pp. 17071716, 2012.

[102] D. Zhao, J. Feng, Q. Huo et al., "Triblock copolymer synthesis of mesoporous silica with periodic 50 to 300 angstrom pores," Science, vol. 279, pp. 548-552, 1998.

[103] D. Zhao, Q. Huo, J. Feng, B. F. Chmelka, and G. D. Stucky, "Nonionic triblock and star diblock copolymer and oligomeric surfactant synthesis of highly ordered, hydrothermally stable, mesoporous silica structures," Journal of the American Chemical Society, vol. 120, pp. 6024-6036, 1998.

[104] J. S. Beck, J. C. Vartuli, W. J. Roth et al., "A new family of mesoporous molecular sieves prepared with liquid crystal templates," Journal of the American Chemical Society, vol. 114, no. 27, pp. 10834-10843, 1992.

[105] A. Y. Khodakov, V. L Zholobenko, M. Impéror-Clerc, and D. Durand, "Characterisation of the initial stages of SBA-15 synthesis by in situ time-resolved X-ray diffraction," The Journal of Physical Chemistry B, vol. 109, no. 48, pp. 22780-22790, 2005.

[106] S. Manet, J. Schmitt, M. Impéror-Clerc et al., "Kinetics of the formation of 2D-hexagonal silica nanostructured materials by nonionic block copolymer templating in solution," Journal of Physical Chemistry B, vol. 115, no. 39, pp. 11330-11344, 2011.

[107] T. Kjellman, N. Reichhardt, M. Sakeye, J.-H. Smaištt, M. Lindén, and V. Alfredsson, "Independent fine-tuning of the intrawall porosity and primary mesoporosity of SBA-15," Chemistry of Materials, vol. 25, no. 9, pp. 1989-1997, 2013.

[108] Y. Wan and D. Zhao, "On the controllable soft-templating approach to mesoporous silicates," Chemical Reviews, vol. 107, no. 7, pp. 2821-2860, 2007.

[109] E. Gianotti, G. Berlier, K. Costabello, S. Coluccia, and F. Meneau, "In situ synchrotron small-angle X-ray scattering study of MCM-41 crystallisation using Gemini surfactants," Catalysis Today, vol. 126, no. 1-2, pp. 203-210, 2007.

[110] M. J. Hollamby, D. Borisova, P. Brown, J. Eastoe, I. Grillo, and D. Shchukin, "Growth of mesoporous silica nanoparticles monitored by time-resolved small-angle neutron scattering," Langmuir, vol. 28, no. 9, pp. 4425-4433, 2012.

[111] L. Bonaccorsi, D. Lombardo, A. Longo, E. Proverbio, and A. Triolo, "Dendrimer template directed self-assembly during zeolite formation," Macromolecules, vol. 42, no. 4, pp. 1239-1243, 2009.

[112] D. Xu, Y. Ma, Z. Jing et al., " $\pi-\pi$ interaction of aromatic groups in amphiphilic molecules directing for single-crystalline mesostructured zeolite nanosheets," Nature Communications, vol. 5, article 4262, 2014.

[113] A. Angelova, B. Angelov, R. Mutafchieva, and S. Lesieur, "Biocompatible mesoporous and soft nanoarchitectures," Journal of Inorganic and Organometallic Polymers and Materials, vol. 25, no. 2, pp. 214-232, 2015.

[114] L. Pasqua, F. Testa, R. Aiello, S. Cundari, and J. B. Nagy, "Preparation of bifunctional hybrid mesoporous silica potentially useful for drug targeting," Microporous and Mesoporous Materials, vol. 103, no. 1-3, pp. 166-173, 2007.

[115] J. Lu, M. Liong, Z. Li, J. I. Zink, and F. Tamanoi, "Biocompatibility, biodistribution, and drug-delivery efficiency of mesoporous silica nanoparticles for cancer therapy in animals," Small, vol. 6, no. 16, pp. 1794-1805, 2010.

[116] C. Morelli, P. Maris, D. Sisci et al., "PEG-templated mesoporous silica nanoparticles exclusively target cancer cells," Nanoscale, vol. 3, no. 8, pp. 3198-3207, 2011.

[117] B. Y. Wang, H. Xu, and X. Zhang, "Tuning the amphiphilicity of building blocks: controlled self-assembly and disassembly for functional supramolecular materials," Advanced Materials, vol. 21, no. 28, pp. 2849-2864, 2009.

[118] M. Marguet, C. Bonduelle, and S. Lecommandoux, "Multicompartmentalized polymeric systems: towards biomimetic cellular structure and function," Chemical Society Reviews, vol. 42, no. 2 , pp. 512-529, 2013.

[119] K. Werengowska-Ciećwierz, M. Wiśniewski, A. P. Terzyk, and S. Furmaniak, "The chemistry of bioconjugation in nanoparticlesbased drug delivery system," Advances in Condensed Matter Physics, vol. 2015, Article ID 198175, 27 pages, 2015.

[120] A. V. Dubtsov, S. V. Pasechnik, D. V. Shmeliova, S. Kralj, and R. Repnik, "Controlled nanoparticle targeting and nanoparticledriven nematic structural transition," Advances in Condensed Matter Physics, vol. 2015, Article ID 803480, 9 pages, 2015.

[121] C. Wang, Z. Wang, and X. Zhang, "Amphiphilic building blocks for self-assembly: from amphiphiles to supra-amphiphiles," Accounts of Chemical Research, vol. 45, no. 4, pp. 608-618, 2012.

[122] H. Zhu, D. Balint, and Z. Guo, "Theoretical analysis and computational simulation of advanced structured materials," Advances in Condensed Matter Physics, vol. 2014, Article ID 367916, 2 pages, 2014.

[123] A. Massoudi, M. Adeli, and L. Khosravi Far, "Fully supramolecular polyrotaxanes as biphase drug delivery systems," International Journal of Polymer Science, vol. 2014, Article ID 829474, 9 pages, 2014.

[124] B. Song, G. Wu, Z. Wang, X. Zhang, M. Smet, and W. Dehaen, "Metal-ligand coordination-induced self-assembly of bolaamphiphiles bearing bipyrimidine," Langmuir, vol. 25, no. 23, pp. 13306-13310, 2009.

[125] G. J. Richards, J. P. Hill, J. Labuta, Y. Wakayama, M. Akada, and K. Ariga, "Self-assembled pyrazinacene nanotubes," Physical Chemistry Chemical Physics, vol. 13, no. 11, pp. 4868-4876, 2011.

[126] Y. W. Huang and Z. X. Wei, "Self-assembly of chiral amphiphiles with $\pi$-conjugated tectons," Chinese Science Bulletin, vol. 57, no. 33, pp. 4246-4256, 2012.

[127] Y. Huang, J. Liu, Y. Cui et al., "Reduction-triggered breakable micelles of amphiphilic polyamide amine-G-polyethylene glycol for methotrexate delivery," BioMed Research International, vol. 2014, Article ID 904634, 11 pages, 2014.

[128] Z. Liu, J. Qiao, Y. Tian, M. Wu, Z. Niu, and Y. Huang, "Polymeric supra-amphiphiles based on terminal group electrostatic interactions: fabrication of micelles with modifiable surfaces," Langmuir, vol. 30, no. 29, pp. 8938-8944, 2014.

[129] J. Wang, X. Wang, F. Yang, H. Shen, Y. You, and D. Wu, "Selfassembly behavior of a linear-star supramolecular amphiphile based on host-guest complexation," Langmuir, vol. 30, no. 43, pp. 13014-13020, 2014.

[130] X. Ji, J. Li, J. Chen et al., "Supramolecular micelles constructed by crown ether-based molecular recognition," Macromolecules, vol. 45, no. 16, pp. 6457-6463, 2012.

[131] G. Wang, Y. Kang, B. Tang, and X. Zhang, “Tuning the surface activity of gemini amphiphile by the host-guest interaction of cucurbit[7]uril," Langmuir, vol. 31, no. 1, pp. 120-124, 2015. 
[132] S. B. Nimse and T. Kim, "Biological applications of functionalized calixarenes," Chemical Society Reviews, vol. 42, no. 1, pp. 366-386, 2013.

[133] C. Y. Ang, S. Y. Tan, X. Wang et al., "Supramolecular nanoparticle carriers self-assembled from cyclodextrin- and adamantanefunctionalized polyacrylates for tumor-targeted drug delivery," Journal of Materials Chemistry B, vol. 2, no. 13, pp. 1879-1890, 2014.

[134] J. Deng, X. Liu, W. Shi, C. Cheng, C. He, and C. Zhao, "Lighttriggered switching of reversible and alterable biofunctionality via $\beta$-cyclodextrin/azobenzene-based host-guest interaction," ACS Macro Letters, vol. 3, no. 11, pp. 1130-1133, 2014.

[135] G. Zhang, F. Hu, and D. Zhang, "Manipulation of the aggregation and deaggregation of tetraphenylethylene and silole fluorophores by amphiphiles: emission modulation and sensing applications," Langmuir, vol. 31, no. 16, pp. 4593-4604, 2014.

[136] D. Luo, X.-P. Zhou, and D. Li, "Beyond molecules: mesoporous supramolecular frameworks self-assembled from coordination cages and inorganic anions," Angewandt Chemie-International Edition, vol. 127, no. 21, pp. 6288-6293, 2015.

[137] N. Ahmad, H. A. Younus, A. H. Chughtai, and F. Verpoort, "Metal-organic molecular cages: applications of biochemical implications," Chemical Society Reviews, vol. 44, no. 1, pp. 9-25, 2015.

[138] N. Ahmad, A. H. Chughtai, H. A. Younus, and F. Verpoort, "Discrete metal-carboxylate self-assembled cages: design, synthesis and applications," Coordination Chemistry Reviews, vol. 280, pp. 1-27, 2014.

[139] T. R. Cook and P. J. Stang, "Recent developments in the preparation and chemistry of metallacycles and metallacages via coordination," Chemical Reviews, vol. 115, no. 15, pp. 7001-7045, 2015. 

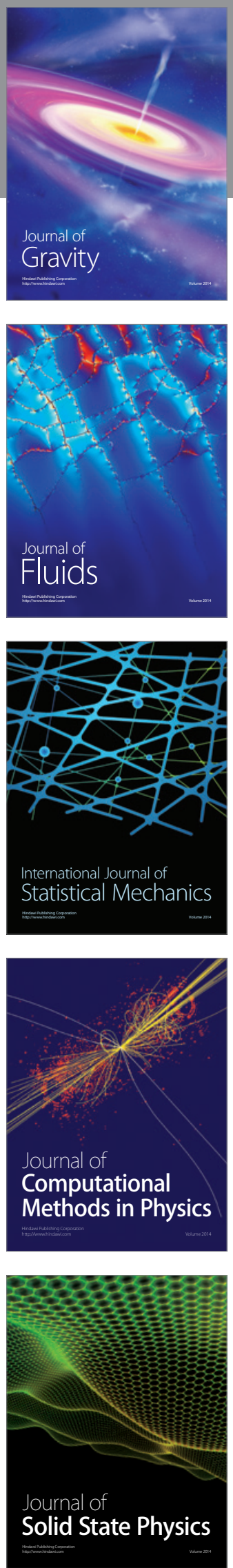

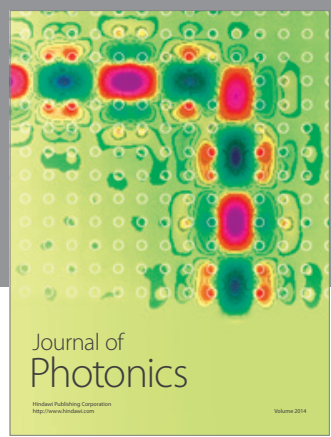

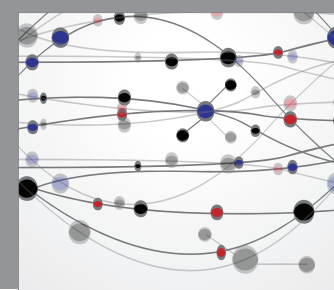

The Scientific World Journal

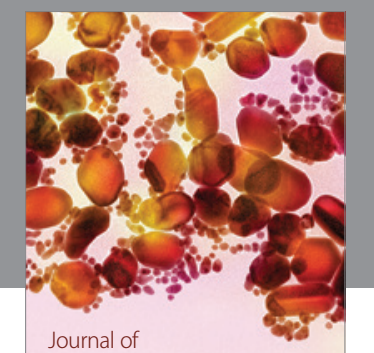

Soft Matter
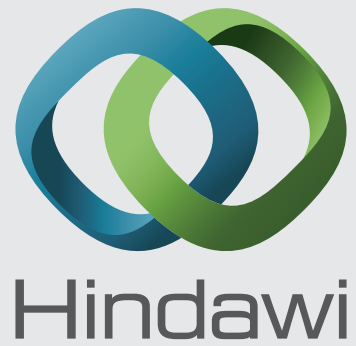

Submit your manuscripts at

http://www.hindawi.com
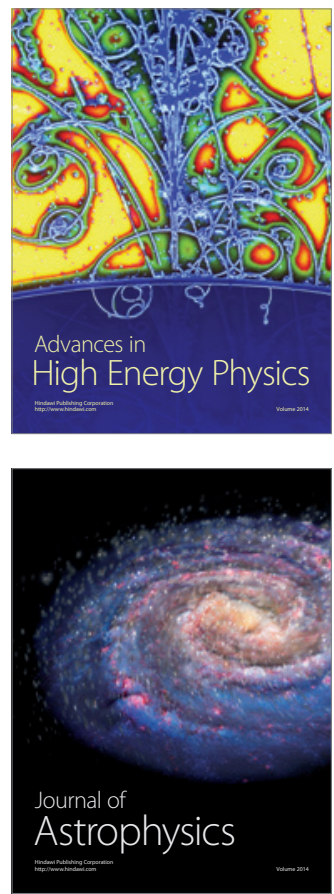
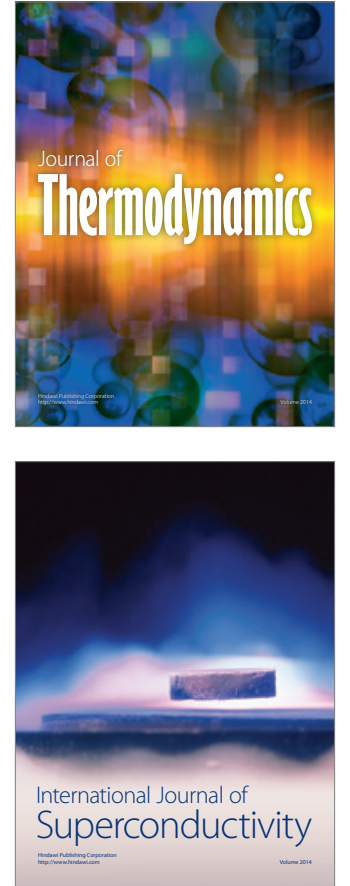
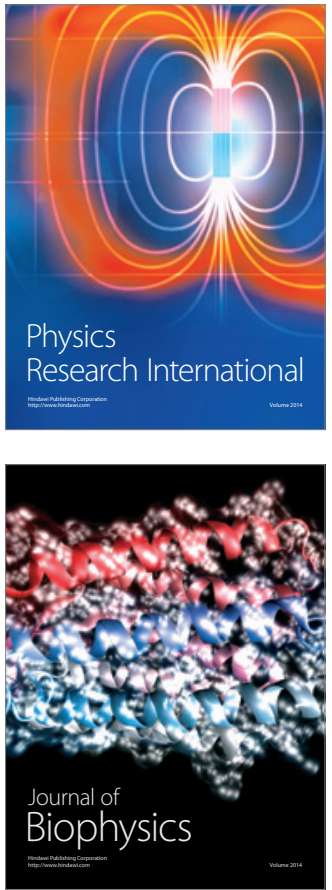
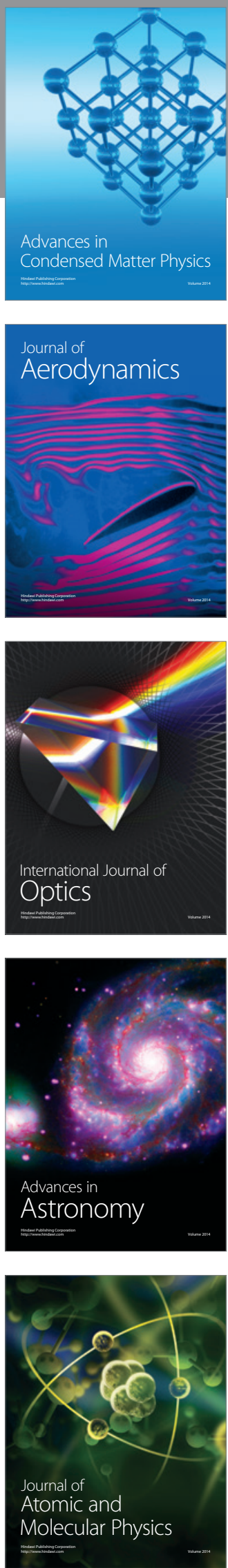